\title{
DYNAMICAL PROPERTIES OF FAMILIES OF HOLOMORPHIC MAPPINGS
}

\author{
RATNA PAL AND KAUSHAL VERMA
}

\begin{abstract}
We study some dynamical properties of skew products of Hénon maps of $\mathbb{C}^{2}$ that are fibered over a compact metric space $M$. The problem reduces to understanding the dynamical behavior of the composition of a pseudorandom sequence of Hénon mappings. In analogy with the dynamics of the iterates of a single Hénon map, it is possible to construct fibered Green functions that satisfy suitable invariance properties and the corresponding stable and unstable currents. This analogy is carried forth in two ways: it is shown that the successive pull-backs of a suitable current by the skew Hénon maps converges to a multiple of the fibered stable current and secondly, this convergence result is used to obtain a lower bound on the topological entropy of the skew product in some special cases. The other class of maps that are studied are skew products of holomorphic endomorphisms of $\mathbb{P}^{k}$ that are again fibered over a compact base. We define the fibered Fatou component and show that they are pseudoconvex and Kobayashi hyperbolic.
\end{abstract}

\section{INTRODUCTION}

The purpose of this note is to study various dynamical properties of some classes of fibered mappings. We will first consider families of the form $H: M \times \mathbb{C}^{2} \rightarrow M \times \mathbb{C}^{2}$ defined by

$$
H(\lambda, x, y)=\left(\sigma(\lambda), H_{\lambda}(x, y)\right)
$$

where $M$ is an appropriate parameter space, $\sigma$ is a self map of $M$ and for each $\lambda \in M$, the map

$$
H_{\lambda}(x, y)=H_{\lambda}^{(m)} \circ H_{\lambda}^{(m-1)} \circ \cdots \circ H_{\lambda}^{(1)}(x, y)
$$

where for every $1 \leq j \leq m$,

$$
H_{\lambda}^{(j)}(x, y)=\left(y, p_{j, \lambda}(y)-a_{j}(\lambda) x\right)
$$

is a generalized Hénon map with $p_{j, \lambda}(y)$ a monic polynomial of degree $d_{j} \geq 2$ whose coefficients and $a_{j}(\lambda)$ are functions on $M$. The degree of $H_{\lambda}$ is $d=d_{1} d_{2} \cdots d_{m}$ which does not vary with $\lambda$. The two cases that will be considered here are as follows. First, $M$ is a compact metric space and $\sigma, a_{j}$ and the coefficients of $p_{j, \lambda}$ are continuous functions on $M$ and second, $M \subset \mathbb{C}^{k}, k \geq 1$ is open in which case $\sigma$, $a_{j}$ and the coefficients of $p_{j, \lambda}$ are assumed to be holomorphic in $\lambda$. In both cases, $a_{j}$ is assumed to be a non-vanishing function on $M$. We are interested in studying the ergodic properties of such a family of mappings. Part of the reason for this choice

Received by the editors April 22, 2015 and, in revised form, September 28, 2015.

2010 Mathematics Subject Classification. Primary 37F45.

The first named author was supported by CSIR-UGC (India) fellowship.

The second named author was supported by the DST Swarna Jayanti Fellowship 2009-2010 and a UGC-CAS Grant. 
stems from the Fornaess-Wu classification ([12]) of polynomial automorphisms of $\mathbb{C}^{3}$ of degree at most 2 according to which any such map is affinely conjugate to

(a) an affine automorphism,

(b) an elementary polynomial automorphism of the form

$$
E(x, y, z)=(P(y, z)+a x, Q(z)+b y, c z+d)
$$

where $P, Q$ are polynomials with $\max \{\operatorname{deg}(P), \operatorname{deg}(Q)\}=2$ and $a b c \neq 0$ or (c) to one of the following:

- $H_{1}(x, y, z)=(P(x, z)+a y, Q(z)+x, c z+d)$,

- $H_{2}(x, y, z)=(P(y, z)+a x, Q(y)+b z, y)$,

- $H_{3}(x, y, z)=(P(x, z)+a y, Q(x)+z, x)$,

- $H_{4}(x, y, z)=(P(x, y)+a z, Q(y)+x, y)$,

- $H_{5}(x, y, z)=(P(x, y)+a z, Q(x)+b y, x)$,

where $P, Q$ are polynomials with $\max \{\operatorname{deg}(P), \operatorname{deg}(Q)\}=2$ and $a b c \neq 0$.

The six classes in (b) and (c) together were studied in [4] and [5] where suitable Green functions and associated invariant measures were constructed for them. As observed in 12, several maps in (c) are in fact families of Hénon maps for special values of the parameters $a, b, c$ and for judicious choices of the polynomials $P, Q$. For instance, if $Q(z)=0$ and $P(x, z)=x^{2}+\cdots$, then $H_{1}(x, y, z)=(P(x, z)+a y, x, z)$ which is conjugate to

$$
(x, y, z) \mapsto(y, P(y, z)+a x, c z+d)=\left(y, y^{2}+\cdots+a x, c z+d\right)
$$

by the inversion $\tau_{1}(x, y, z)=(y, x, z)$. Here $\sigma(z)=c z+d$. Similarly, if $a=1$, $P(y, z)=0$ and $Q$ is a quadratic polynomial, then $H_{2}(x, y, z)=(x, Q(y)+b z, y)$ which is conjugate to

$$
(x, y, z) \mapsto(x, z, Q(z)+b y)=\left(x, z, z^{2}+\cdots+b y\right)
$$

by the inversion $\tau_{3}(x, y, z)=(x, z, y)$. Here $\sigma(x)=x$ and finally, if $b=1, Q(x)=0$ and $P(x, y)=x^{2}+\cdots$, then $H_{5}(x, y, z)=(P(x, y)+a z, y, x)$ which is conjugate to

$$
(x, y, z) \mapsto(z, y, P(z, y)+a x)=\left(z, y, z^{2}+\cdots+a x\right)
$$

by the inversion $\tau_{5}(x, y, z)=(z, y, x)$ where again $\sigma(y)=y$. All of these are examples of the kind described in (1.1) with $M=\mathbb{C}$. In the first example, if $c \neq 1$, then an affine change of coordinates involving only the $z$-variable can make $d=0$ and if further $|c| \leq 1$, then we may take a closed disc around the origin in $\mathbb{C}$ which will be preserved by $\sigma(z)=c z$. This provides an example of a Hénon family that is fibered over a compact base $M$. Further, since the parameter mapping $\sigma$ in the last two examples is just the identity, we may restrict it to a closed ball to obtain more examples of the case when $M$ is compact.

The maps considered in (1.1) are in general $q$-regular, for some $q \geq 1$, in the sense of Guedj-Sibony ([14]) as the following example shows. Let $\mathcal{H}: \mathbb{C}^{3} \rightarrow \mathbb{C}^{3}$ be given by

$$
\mathcal{H}(\lambda, x, y)=\left(\lambda, y, y^{2}-a x\right), a \neq 0
$$

which in homogeneous coordinates becomes

$$
\mathcal{H}([\lambda: x: y: t])=\left[\lambda t: y t: y^{2}-a x t: t^{2}\right] .
$$

The indeterminacy set of this map is $I^{+}=[\lambda: x: 0: 0]$ while that for $\mathcal{H}^{-1}$ is $I^{-1}=[\lambda: 0: y: 0]$. Thus $I^{+} \cap I^{-}=[1: 0: 0: 0]$ and it can be checked that $X^{+}=\overline{\mathcal{H}\left((t=0) \backslash I^{+}\right)}=[0: 0: 1: 0]$ which is disjoint from $I^{+}$. Also, $X^{-}=$ 
$\overline{\mathcal{H}^{-}\left((t=0) \backslash I^{-}\right)}=[0: 1: 0: 0]$ which is disjoint from $I^{-}$. All these observations imply that $\mathcal{H}$ is 1 -regular in the sense of [14. Further, $\operatorname{deg}(\mathcal{H})=\operatorname{deg}\left(\mathcal{H}^{-1}\right)=2$. This global view point does have several advantages as the results in [14, 13. show. However, thinking of (1.1) as a family of maps was seconded by the hope that the methods of Bedford-Smillie ([1], 2] and [3]) and Fornaess-Sibony [1] that were developed to handle the case of a single generalized Hénon map would be amenable to this situation; in fact, they are to a large extent. Finally, in view of the systematic treatment of families of rational maps of the sphere by Jonsson (see [16], [17]), considering families of Hénon maps appeared to be a natural next choice. Several pertinent remarks about the family $H$ in (1.1) with $\sigma(\lambda)=\lambda$ can be found in $[8$.

Let us first work with the case when $M$ is a compact metric space. For $n \geq 0$, let

$$
H_{\lambda}^{ \pm n}=H_{\sigma^{n-1}(\lambda)}^{ \pm 1} \circ \cdots \circ H_{\sigma(\lambda)}^{ \pm 1} \circ H_{\lambda}^{ \pm 1}
$$

Note that $H_{\lambda}^{+n}$ is the second coordinate of the $n$-fold iterate of $H(\lambda, x, y)$. Furthermore,

$$
\left(H_{\lambda}^{+n}\right)^{-1}=H_{\lambda}^{-1} \circ H_{\sigma(\lambda)}^{-1} \circ \cdots \circ H_{\sigma^{n-1}(\lambda)}^{-1} \neq H_{\lambda}^{-n}
$$

and

$$
\left(H_{\lambda}^{-n}\right)^{-1}=H_{\lambda} \circ H_{\sigma(\lambda)} \circ \cdots \circ H_{\sigma^{n-1}(\lambda)} \neq H_{\lambda}^{+n}
$$

for $n \geq 2$. The presence of $\sigma$ creates an asymmetry which is absent in the case of a single Hénon map and which requires the consideration of these maps as will be seen later. In what follows, no conditions on $\sigma$ except continuity are assumed unless stated otherwise.

While the essence of the proofs in what follows remains the same as in the classical case of a single Hénon map, it must be emphasized that there are several technical and non-trivial modifications that need to be consistently made throughout this entire analysis. To reiterate, this happens due to the presence of the asymmetry introduced by the skew map $\sigma$ which makes the global map $H$ non-invertible. Starting with the existence of a uniform filtration, to the various properties of the Green functions and then to the convergence theorem for currents in which different Hénon maps are used to pull-back a given current, each step requires an additional input on top of what exists in the well studied classical case.

The first thing to do is to construct invariant measures for the family $H(\lambda, x, y)$ that respect the action of $\sigma$. The essential step toward this is to construct a uniform filtration $V_{R}, V_{R}^{ \pm}$for the maps $H_{\lambda}$ where $R>0$ is sufficiently large.

For each $\lambda \in M$, the sets $I_{\lambda}^{ \pm}$of escaping points and the sets $K_{\lambda}^{ \pm}$of non-escaping points under random iteration determined by $\sigma$ on $M$ are defined as follows:

$$
\begin{gathered}
I_{\lambda}^{ \pm}=\left\{z \in \mathbb{C}^{2}:\left\|H_{\lambda}^{ \pm n}(x, y)\right\| \rightarrow \infty \text { as } n \rightarrow \infty\right\}, \\
K_{\lambda}^{ \pm}=\left\{z \in \mathbb{C}^{2}: \text { the sequence }\left\{H_{\lambda}^{ \pm n}(x, y)\right\}_{n} \text { is bounded }\right\} .
\end{gathered}
$$

Clearly, $H_{\lambda}^{ \pm 1}\left(K_{\lambda}^{ \pm}\right)=K_{\sigma(\lambda)}^{ \pm}$and $H_{\lambda}^{ \pm 1}\left(I_{\lambda}^{ \pm}\right)=I_{\sigma(\lambda)}^{ \pm}$. Define $K_{\lambda}=K_{\lambda}^{+} \cap K_{\lambda}^{-}, J_{\lambda}^{ \pm}=$ $\partial K_{\lambda}^{ \pm}$and $J_{\lambda}=J_{\lambda}^{+} \cap J_{\lambda}^{-}$. For each $\lambda \in M$ and $n \geq 1$, let

$$
G_{n, \lambda}^{ \pm}(x, y)=\frac{1}{d^{n}} \log ^{+}\left\|H_{\lambda}^{ \pm n}(x, y)\right\|
$$

where $\log ^{+} t=\max \{\log t, 0\}$. 
Proposition 1.1. The sequence $G_{n, \lambda}^{ \pm}$converges uniformly on compact subsets of $\mathbb{C}^{2}$ to the continuous function $G_{\lambda}^{ \pm}$as $n \rightarrow \infty$ that satisfies

$$
d G_{\lambda}^{ \pm}=G_{\sigma(\lambda)}^{ \pm} \circ H_{\lambda}^{ \pm 1}
$$

on $\mathbb{C}^{2}$. Moreover, the convergence is independent of $\lambda$. The functions $G_{\lambda}^{ \pm}$are positive pluriharmonic on $\mathbb{C}^{2} \backslash K_{\lambda}^{ \pm}$, plurisubharmonic on $\mathbb{C}^{2}$ and vanish precisely on $K_{\lambda}^{ \pm}$. The correspondence $\lambda \mapsto G_{\lambda}^{ \pm}$is continuous. In case $\sigma$ is surjective, $G_{\lambda}^{+}$ is locally uniformly Hölder continuous, i.e., for each compact $S \subset \mathbb{C}^{2}$, there exist constants $\tau, C>0$ such that

$$
\left|G_{\lambda}^{+}(x, y)-G_{\lambda}^{+}\left(x^{\prime}, y^{\prime}\right)\right| \leq C\left\|(x, y)-\left(x^{\prime}, y^{\prime}\right)\right\|^{\tau}
$$

for all $(x, y),\left(x^{\prime}, y^{\prime}\right) \in S$. The constants $\tau, C$ depend on $S$ and the map $H$ only.

As a result, $\mu_{\lambda}^{ \pm}=d d^{c} G_{\lambda}^{ \pm}$are well-defined positive closed $(1,1)$-currents on $\mathbb{C}^{2}$ of mass $2 \pi$ and hence $\mu_{\lambda}=\mu_{\lambda}^{+} \wedge \mu_{\lambda}^{-}$defines a measure of finite mass on $\mathbb{C}^{2}$ whose support is contained in $V_{R}$ for every $\lambda \in M$. While positivity of $\mu_{\lambda}$ follows from the fact that $\mu_{\lambda}^{+}$and $\mu_{\lambda}^{-}$are both positive, the fact that it has mass $4 \pi^{2}$ follows from the next proposition. Moreover, the correspondence $\lambda \mapsto \mu_{\lambda}$ is continuous. That these objects are well behaved under the pull-back and push-forward operations by $H_{\lambda}$ and at the same time respect the action of $\sigma$ is recorded in the following:

Proposition 1.2. With $\mu_{\lambda}^{ \pm}, \mu_{\lambda}$ as above, we have

$$
\left(H_{\lambda}^{ \pm 1}\right)^{*} \mu_{\sigma(\lambda)}^{ \pm}=d \mu_{\lambda}^{ \pm} \text {and }\left(H_{\lambda}^{ \pm 1}\right)_{*} \mu_{\lambda}^{ \pm}=d^{-1} \mu_{\sigma(\lambda)}^{\mp} .
$$

The support of $\mu_{\lambda}^{ \pm}$equals $J_{\lambda}^{ \pm}$and the correspondence $\lambda \mapsto J_{\lambda}^{ \pm}$is lower semicontinuous. Furthermore, for each $\lambda \in M$, the pluricomplex Green function of $K_{\lambda}$ is $\max \left\{G_{\lambda}^{+}, G_{\lambda}^{-}\right\}, \mu_{\lambda}$ is the complex equilibrium measure of $K_{\lambda}, \operatorname{supp}\left(\mu_{\lambda}\right) \subseteq J_{\lambda}$ and $\int_{\mathbb{C}^{2}} \mu_{\lambda}=4 \pi^{2}$.

In particular, if $\sigma$ is the identity on $M$, then $\left(H_{\lambda}^{ \pm 1}\right)^{*} \mu_{\lambda}=\mu_{\lambda}$.

All these generalizations would be rendered artificial if they did not produce something different. To see what happens when a current is pulled back by a parametrized family of Hénon map, let $T$ be a positive closed $(1,1)$-current in a domain $\Omega \subset \mathbb{C}^{2}$ and let $\psi \in C_{0}^{\infty}(\Omega)$ with $\psi \geq 0$ be such that $\operatorname{supp}(\psi) \cap \operatorname{supp}(d T)=$ $\phi$. Further, for each $\lambda \in M$, let $S_{\lambda}(\psi, T)$ be the set of all possible limit points of the sequence $d^{-n}\left(H_{\lambda}^{+n}\right)^{*}(\psi T)$. Then it follows from Theorem 1.3 that $S_{\lambda}(\psi, T)$ may contain several limit points, all of which are multiples of $\mu_{\lambda}^{+}$. In the classical case, this set consists of precisely a single limit point. In fact, Theorem 1.6 in 3 ] shows that for a single Hénon map $H$ of degree $d$, the sequence $d^{-n} H^{n *}(\psi T)$ always converges to $c \mu^{+}$where $c=\frac{1}{4 \pi^{2}} \int \psi T \wedge \mu^{-}>0$.

Theorem 1.3. Let $T$ and $\psi$ be as above. Then for all $\lambda \in M, S_{\lambda}(\psi, T)$ is nonempty and each $\gamma_{\lambda} \in S_{\lambda}(\psi, T)$ is a positive multiple of $\mu_{\lambda}^{+}$.

In general, $S_{\lambda}(\psi, T)$ may be a large set and this is the main new phenomenon that is encountered while dealing with a parametrized family of Hénon maps. However, there are two cases for which it is possible to determine the cardinality of $S_{\lambda}(\psi, T)$ and both are illustrated by the examples mentioned earlier.

Proposition 1.4. If $\sigma$ is the identity on $M$ or when $\sigma: M \rightarrow M$ is a contraction, i.e., there exists $\lambda_{0} \in M$ such that $\sigma^{n}(\lambda) \rightarrow \lambda_{0}$ for all $\lambda \in M$, the set $S_{\lambda}(\psi, T)$ 
consists of precisely one element. Consequently, in each of these cases there exists a constant $c_{\lambda}(\psi, T)>0$ such that

$$
\lim _{n \rightarrow \infty} d^{-n}\left(H_{\lambda}^{+n}\right)^{*}(\psi T)=c_{\lambda}(\psi, T) \mu_{\lambda}^{+} .
$$

Let us now consider the case when $M$ is a relatively compact open subset of $\mathbb{C}^{k}, k \geq 1$ and the map $\sigma$ is the identity on $M$. Since this means that the slices over each point in $M$ are preserved, we may (by shrinking $M$ slightly) assume that the maps $H_{\lambda}$ are well defined in a neighborhood of $\bar{M}$. Thus the earlier discussion about the construction of $\mu_{\lambda}^{ \pm}, \mu_{\lambda}$ applies to the family (which will henceforth be considered)

$$
\begin{gathered}
H: M \times \mathbb{C}^{2} \rightarrow M \times \mathbb{C}^{2}, \\
H(\lambda, x, y)=\left(\lambda, H_{\lambda}(x, y)\right) .
\end{gathered}
$$

For every probability measure $\mu^{\prime}$ on $M$,

$$
\langle\mu, \varphi\rangle=\frac{1}{4 \pi^{2}} \int_{M}\left(\int_{\{\lambda\} \times \mathbb{C}^{2}} \varphi \mu_{\lambda}\right) \mu^{\prime}(\lambda)
$$

defines a measure on $M \times \mathbb{C}^{2}$ by describing its action on continuous functions $\varphi$ on $M \times \mathbb{C}^{2}$. This is not a dynamically natural measure since $\mu^{\prime}$ is arbitrary. It will turn out that the support of $\mu$ is contained in

$$
\mathcal{J}=\overline{\bigcup_{\lambda \in M}\left(\{\lambda\} \times J_{\lambda}\right)} \subset M \times V_{R} .
$$

The slice measures of $\mu$ are evidently $\mu_{\lambda}$ and since $\sigma$ is the identity it can be seen from Proposition 1.2 that $\mu$ is an invariant probability measure for $H$ as above.

Theorem 1.5. Regard $H$ as a self map of $\operatorname{supp}(\mu)$ with invariant probability measure $\mu$. The measure theoretic entropy of $H$ with respect to $\mu$ is at least $\log d$. In particular, the topological entropy of $H: \mathcal{J} \rightarrow \mathcal{J}$ is at least $\log d$.

The above theorem strengthens the lower bound of the topological entropy of a single Hénon map obtained by J. Smillie in [21 and a proof of Theorem 1.5] would also follow from a parametrized version of his arguments. However, we have chosen to follow the approach of Bedford-Smillie ([3] ) since it provides information about the measure theoretic entropy with respect to the measure $\mu$ (which involves an arbitrary $\mu^{\prime}$ ) as in (1.2).

It would be both interesting and desirable to obtain lower bounds for the topological entropy for an arbitrary continuous function $\sigma$ in (1.1).

Finally, the discerning reader will surely ask whether some of these results carry over to the case of a compact family of Hénon maps, i.e., a family that is not necessarily parametrized by $\sigma$. In fact, this is true but the proofs are necessarily different from the ones presented here and so are the applications that we have in mind. These would fall within the paradigm of random dynamics in the spirit of Fornaess-Weickert [12] (for example) and others; for this reason, the details of such a study would be presented elsewhere in the near future.

We will now consider continuous families of holomorphic endomorphisms of $\mathbb{P}^{k}$. For a compact metric space $M, \sigma$ a continuous self map of $M$, define $F: M \times \mathbb{P}^{k} \rightarrow$ $M \times \mathbb{P}^{k}$ as

$$
F(\lambda, z)=\left(\sigma(\lambda), f_{\lambda}(z)\right)
$$


where $f_{\lambda}$ is a holomorphic endomorphism of $\mathbb{P}^{k}$ that depends continuously on $\lambda$. Each $f_{\lambda}$ is assumed to have a fixed degree $d \geq 2$. Corresponding to each $f_{\lambda}$ there exists a non-degenerate homogeneous holomorphic mapping $F_{\lambda}: \mathbb{C}^{k+1} \rightarrow \mathbb{C}^{k+1}$ such that $\pi \circ F_{\lambda}=f_{\lambda} \circ \pi$ where $\pi: \mathbb{C}^{k+1} \backslash\{0\} \rightarrow \mathbb{P}^{k}$ is the canonical projection. Here, non-degeneracy means that $F_{\lambda}^{-1}(0)=0$ which in turn implies that there are uniform constants $l, L>0$ with

$$
l\|x\|^{d} \leq\left\|F_{\lambda}(x)\right\| \leq L\|x\|^{d}
$$

for all $\lambda \in M$ and $x \in \mathbb{C}^{k+1}$. Therefore, for $0<r \leq(2 L)^{-1 /(d-1)}$

$$
\left\|F_{\lambda}(x)\right\| \leq(1 / 2)\|x\|
$$

for all $\lambda \in M$ and $\|x\| \leq r$. Likewise, for $R \geq(2 l)^{-1 /(d-1)}$,

$$
\left\|F_{\lambda}(x)\right\| \geq 2\|x\|
$$

for all $\lambda \in M$ and $\|x\| \geq R$.

While the ergodic properties of such a family have been considered in [6], [7], for instance, we are interested in looking at the basins of attraction which may be defined for each $\lambda \in M$ as

$$
\mathcal{A}_{\lambda}=\left\{x \in \mathbb{C}^{k+1}: F_{\sigma^{n-1}(\lambda)} \circ \cdots \circ F_{\sigma(\lambda)} \circ F_{\lambda}(x) \rightarrow 0 \text { as } n \rightarrow \infty\right\}
$$

and for each $\lambda \in M$, the region of normality $\Omega_{\lambda}^{\prime} \subset \mathbb{P}^{k}$ which consists of all points $z \in \mathbb{P}^{k}$ for which there is a neighborhood $V_{z}$ on which the sequence $\left\{f_{\sigma^{n-1}(\lambda)} \circ \cdots \circ\right.$ $\left.f_{\sigma(\lambda)} \circ f_{\lambda}\right\}_{n \geq 1}$ is normal. Analogs of $\mathcal{A}_{\lambda}$ arising from composing a given sequence of automorphisms of $\mathbb{C}^{n}$ have been considered in [19] where an example can be found for which these are not open in $\mathbb{C}^{n}$. However, since each $F_{\lambda}$ is homogeneous, it is straightforward to verify that each $\mathcal{A}_{\lambda}$ is a non-empty, pseudoconvex complete circular domain. As in the case of a single holomorphic endomorphism of $\mathbb{P}^{k}$ (see [15], 22]), the link between these two domains is provided by the Green function.

For each $\lambda \in M$ and $n \geq 1$, let

$$
G_{n, \lambda}(x)=\frac{1}{d^{n}} \log \left\|F_{\sigma^{n-1}(\lambda)} \circ \cdots \circ F_{\sigma(\lambda)} \circ F_{\lambda}(x)\right\| .
$$

Proposition 1.6. For each $\lambda \in M$, the sequence $G_{n, \lambda}$ converges uniformly on $\mathbb{C}^{k+1}$ to a continuous plurisubharmonic function $G_{\lambda}$ which satisfies

$$
G_{\lambda}(c x)=\log |c|+G_{\lambda}(x)
$$

for $c \in \mathbb{C}^{*}$. Further, $d G_{\lambda}=G_{\sigma(\lambda)} \circ F_{\lambda}$, and $G_{\lambda_{n}} \rightarrow G_{\lambda}$ locally uniformly on $\mathbb{C}^{k+1} \backslash\{0\}$ as $\lambda_{n} \rightarrow \lambda$ in $M$. Finally,

$$
\mathcal{A}_{\lambda}=\left\{x \in \mathbb{C}^{k+1}: G_{\lambda}(x)<0\right\}
$$

for each $\lambda \in M$.

For each $\lambda \in M$, let $\mathcal{Q}_{\lambda} \subset \mathbb{C}^{k+1}$ be the collection of those points in a neighborhood of which $G_{\lambda}$ is pluriharmonic and define $\Omega_{\lambda}=\pi\left(\mathcal{Q}_{\lambda}\right) \subset \mathbb{P}^{k}$.

Proposition 1.7. For each $\lambda \in M, \Omega_{\lambda}=\Omega_{\lambda}^{\prime}$. Further, each $\Omega_{\lambda}$ is pseudoconvex and Kobayashi hyperbolic. 


\section{Fibered families of HÉnOn maps}

The existence of a filtration $V_{R}^{ \pm}, V_{R}$ for a Hénon map is useful in localizing its dynamical behavior. To study a family of such maps, it is therefore essential to first establish the existence of a uniform filtration that works for all of them. Let

$$
\begin{aligned}
& V_{R}^{+}=\left\{(x, y) \in \mathbb{C}^{2}:|y|>|x|,|y|>R\right\}, \\
& V_{R}^{-}=\left\{(x, y) \in \mathbb{C}^{2}:|y|<|x|,|x|>R\right\} \text { and } \\
& V_{R}=\left\{(x, y) \in \mathbb{C}^{2}:|x|,|y| \leq R\right\}
\end{aligned}
$$

be a filtration of $\mathbb{C}^{2}$ where $R$ is large enough so that

$$
H_{\lambda}\left(V_{R}^{+}\right) \subset V_{R}^{+}
$$

for each $\lambda \in M$. The existence of such an $R$ is shown in the following lemma.

Lemma 2.1. There exists $R>0$ such that

$$
H_{\lambda}\left(V_{R}^{+}\right) \subset V_{R}^{+}, \quad H_{\lambda}\left(V_{R}^{+} \cup V_{R}\right) \subset V_{R}^{+} \cup V_{R}
$$

and

$$
H_{\lambda}^{-1}\left(V_{R}^{-}\right) \subset V_{R}^{-}, \quad H_{\lambda}^{-1}\left(V_{R}^{-} \cup V_{R}\right) \subset V_{R}^{-} \cup V_{R}
$$

for all $\lambda \in M$. Furthermore,

$$
I_{\lambda}^{ \pm}=\mathbb{C}^{2} \backslash K_{\lambda}^{ \pm}=\bigcup_{n=0}^{\infty}\left(H_{\lambda}^{ \pm n}\right)^{-1}\left(V_{R}^{ \pm}\right) .
$$

Proof. Let

$$
p_{j, \lambda}(y)=y^{d_{j}}+c_{\lambda\left(d_{j}-1\right)} y^{d_{j}-1}+\cdots+c_{\lambda 1} y+c_{\lambda 0}
$$

be the polynomial that occurs in the definition of $H_{\lambda}^{(j)}$. Then

$$
\left|y^{-d_{j}} p_{j, \lambda}(y)-1\right| \leq\left|c_{\lambda\left(d_{j}-1\right)} y^{-1}\right|+\cdots+\left|c_{\lambda 1} y^{-d_{j}+1}\right|+\left|c_{\lambda 0} y^{-d_{j}}\right| .
$$

Let $a=\sup _{\lambda, j}\left|a_{\lambda, j}\right|$. Since the coefficients of $p_{j, \lambda}$ are continuous on $M$, which is assumed to be compact, and for $d_{j} \geq 2$ it follows that there exists $R>0$ such that

$$
\left|p_{j, \lambda}(y)\right| \geq(2+a)|y|
$$

for $|y|>R, \lambda \in M$ and $1 \leq j \leq m$. To see that $H_{\lambda}\left(V_{R}^{+}\right) \subset V_{R}^{+}$for this $R$, pick $(x, y) \in V_{R}^{+}$. Then

$$
\left|p_{j, \lambda}(y)-a_{j}(\lambda) x\right| \geq\left|p_{j, \lambda}(y)\right|-\left|a_{j}(\lambda) x\right| \geq|y|
$$

for all $1 \leq j \leq m$. It follows that the second coordinate of each $H_{\lambda}^{(j)}$ dominates the first one. This implies that

$$
H_{\lambda}\left(V_{R}^{+}\right) \subset V_{R}^{+}
$$

for all $\lambda \in M$. The other invariance properties follow by using similar arguments.

Let $\rho>1$ be such that

$$
\left|p_{j, \lambda}(y)-a_{j}(\lambda) x\right|>\rho|y|
$$

for $(x, y) \in \overline{V_{R}^{+}}, \lambda \in M$ and $1 \leq j \leq m$. That a $\rho$ exists follows from (2.2). By letting $\pi_{1}$ and $\pi_{2}$ be the projections on the first and second coordinate respectively, one can conclude inductively that

$$
H_{\lambda}(x, y) \in V_{R}^{+} \text {and }\left|\pi_{2}\left(H_{\lambda}(x, y)\right)\right|>\rho^{m}|y| \text {. }
$$


Analogously, for all $(x, y) \in \overline{V_{R}^{-}}$and for all $\lambda \in M$, there exists a $\rho>1$ satisfying

$$
H_{\lambda}^{-1}(x, y) \in V_{R}^{-} \text {and }\left|\pi_{1}\left(H_{\lambda}^{-1}(x, y)\right)\right|>\rho^{m}|x| .
$$

These two facts imply that

$$
\overline{V_{R}^{+}} \subset H_{\lambda}^{-1}\left(\overline{V_{R}^{+}}\right) \subset H_{\lambda}^{-1} \circ H_{\sigma(\lambda)}^{-1}\left(\overline{V_{R}^{+}}\right) \subset \cdots \subset\left(H_{\lambda}^{+n}\right)^{-1}\left(\overline{V_{R}^{+}}\right) \subset \cdots
$$

and

$$
\overline{V_{R}^{-}} \supset H_{\lambda}^{-1}\left(\overline{V_{R}^{-}}\right) \supset H_{\lambda}^{-1} \circ H_{\sigma(\lambda)}^{-1}\left(\overline{V_{R}^{-}}\right) \supset \cdots \supset\left(H_{\lambda}^{+n}\right)^{-1}\left(\overline{V_{R}^{-}}\right) \supset \cdots .
$$

At this point one can observe that if we start with a point in $\overline{V_{R}^{+}}$, it eventually escapes toward the point at infinity under forward iteration determined by the continuous function $\sigma$, i.e., $\left|H_{\lambda}^{+n}(x, y)\right| \rightarrow \infty$ as $n \rightarrow \infty$. This can be justified by using (2.3) and observing that

$$
\left|y_{\lambda}^{n}\right|>\rho^{m}\left|y_{\lambda}^{n-1}\right|>\rho^{2 m}\left|y_{\lambda}^{n-2}\right|>\cdots>\rho^{n m}|y|>\rho^{n m} R
$$

where $H_{\lambda}^{+n}(x, y)=\left(x_{\lambda}^{n}, y_{\lambda}^{n}\right)$. A similar argument shows that if we start with any point in $(x, y) \in \bigcup_{n=0}^{\infty}\left(H_{\lambda}^{+n}\right)^{-1}\left(V_{R}^{+}\right)$, the orbit of the point never remains bounded. Therefore,

$$
\bigcup_{n=0}^{\infty}\left(H_{\lambda}^{+n}\right)^{-1}\left(V_{R}^{+}\right) \subseteq I_{\lambda}^{+} .
$$

Moreover, using (2.3) and (2.4), we get

$$
\left(H_{\lambda}^{-n}\right)^{-1}\left(V_{R}^{+}\right) \subseteq\left\{(x, y):|y|>\rho^{n m} R\right\}
$$

and

$$
\left(H_{\lambda}^{+n}\right)^{-1}\left(V_{R}^{-}\right) \subseteq\left\{(x, y):|x|>\rho^{n m} R\right\}
$$

which give

$$
\bigcap_{n=0}^{\infty}\left(H_{\lambda}^{-n}\right)^{-1}\left(V_{R}^{+}\right)=\bigcap_{n=0}^{\infty}\left(H_{\lambda}^{-n}\right)^{-1}\left(\overline{V_{R}^{+}}\right)=\phi
$$

and

$$
\bigcap_{n=0}^{\infty}\left(H_{\lambda}^{+n}\right)^{-1}\left(V_{R}^{-}\right)=\bigcap_{n=0}^{\infty}\left(H_{\lambda}^{+n}\right)^{-1}\left(\overline{V_{R}^{-}}\right)=\phi,
$$

respectively. Set

$$
W_{R}^{+}=\mathbb{C}^{2} \backslash \overline{V_{R}^{-}} \text {and } W_{R}^{-}=\mathbb{C}^{2} \backslash \overline{V_{R}^{+}} .
$$

Note that (2.6) and (2.9) are equivalent to

$$
W_{R}^{+} \subset H_{\lambda}^{-1}\left(W_{R}^{+}\right) \subset \cdots \subset\left(H_{\lambda}^{+n}\right)^{-1}\left(W_{R}^{+}\right) \subset \cdots
$$

and

$$
\bigcup_{n=0}^{\infty}\left(H_{\lambda}^{+n}\right)^{-1}\left(W_{R}^{+}\right)=\mathbb{C}^{2},
$$

respectively. Now (2.11) implies that for any point $(x, y) \in \mathbb{C}^{2}$, there exists $n_{0}>0$ such that $H_{\lambda}^{+n}(x, y) \in W_{R}^{+} \subset V_{R} \cup \overline{V_{R}^{+}}$for all $n \geq n_{0}$. So either

$$
H_{\lambda}^{+n}(x, y) \in V_{R}
$$


for all $n \geq n_{0}$ or there exists $n_{1} \geq n_{0}$ such that $H_{\lambda}^{+n_{1}}(x, y) \in \overline{V_{R}^{+}}$. In the latter case, $H_{\lambda}^{+\left(n_{1}+1\right)}(x, y) \in V_{R}^{+}$by (2.3). This implies that

$$
I_{\lambda}^{+}=\mathbb{C}^{2} \backslash K_{\lambda}^{+}=\bigcup_{n=0}^{\infty}\left(H_{\lambda}^{+n}\right)^{-1}\left(V_{R}^{+}\right) .
$$

A set of similar arguments yield

$$
I_{\lambda}^{-}=\mathbb{C}^{2} \backslash K_{\lambda}^{-}=\bigcup_{n=0}^{\infty}\left(H_{\lambda}^{-n}\right)^{-1}\left(V_{R}^{-}\right) .
$$

Remark 2.2. It follows from Lemma 2.1 that for any compact $A_{\lambda} \subset \mathbb{C}^{2}$ satisfying $A_{\lambda} \cap K_{\lambda}^{+}=\phi$, there exists $N_{\lambda}>0$ such that $H_{\lambda}^{+n_{\lambda}}\left(A_{\lambda}\right) \subseteq V_{R}^{+}$. More generally, for any compact $A \subset \mathbb{C}^{2}$ that satisfies $A \cap K_{\lambda}^{+}=\phi$ for each $\lambda \in M$, there exists $N>0$ so that $H_{\lambda}^{+N}(A) \subseteq V_{R}^{+}$for all $\lambda \in M$. The proof again relies on the fact that the coefficients of $p_{j, \lambda}$ and $a_{j}(\lambda)$ vary continuously in $\lambda$ on the compact set $M$ for all $1 \leq j \leq m$.

Remark 2.3. By applying the same kind of techniques as in the case of a single Hénon map, it is possible to show that $I_{\lambda}^{ \pm}$are non-empty, pseudoconvex domains and $K_{\lambda}^{ \pm}$are closed sets satisfying $K_{\lambda}^{ \pm} \cap V_{R}^{ \pm}=\phi$ and having non-empty intersection with the $y$-axis and $x$-axis, respectively. In particular, $K_{\lambda}^{ \pm}$are non-empty and unbounded.

\subsection{Proof of Proposition 1.1.}

Proof. Since the polynomials $p_{j, \lambda}$ are all monic, it follows that for every small $\epsilon_{1}>0$ there is a large enough $R>1$ so that for all $(x, y) \in \overline{V_{R}^{+}}, 1 \leq j \leq m$ and for all $\lambda \in M$, we have $H_{\lambda}^{(j)}(x, y) \in V_{R}^{+}$and

$$
\left(1-\epsilon_{1}\right)|y|^{d_{j}}<\left|\pi_{2} \circ H_{\lambda}^{(j)}(x, y)\right|<\left(1+\epsilon_{1}\right)|y|^{d_{j}} .
$$

For a given $\epsilon>0$, choose $\epsilon_{1}>0$ small enough so that the constants

$$
A_{1}=\prod_{j=1}^{m}\left(1-\epsilon_{1}\right)^{d_{j+1} \cdots d_{m}} \text { and } A_{2}=\prod_{j=1}^{m}\left(1+\epsilon_{1}\right)^{d_{j+1} \cdots d_{m}}
$$

(where $d_{j+1} \cdots d_{m}=1$ by definition when $j=m$ ) satisfy $1-\epsilon \leq A_{1}$ and $A_{2} \leq 1+\epsilon$. Therefore, by applying (2.12) inductively, we get

$$
(1-\epsilon)|y|^{d} \leq A_{1}|y|^{d}<\left|\pi_{2} \circ H_{\lambda}(x, y)\right|<A_{2}|y|^{d} \leq(1+\epsilon)|y|^{d}
$$

for all $\lambda \in M$ and for all $(x, y) \in \overline{V_{R}^{+}}$. Let $(x, y) \in \overline{V_{R}^{+}}$. In view of (2.3) there exists a large $R>1$ so that $H_{\lambda}^{+n}(x, y)=\left(x_{\lambda}^{n}, y_{\lambda}^{n}\right) \in V_{R}^{+}$for all $n \geq 1$ and for all $\lambda \in M$. Therefore,

$$
G_{n, \lambda}^{+}(x, y)=\frac{1}{d^{n}} \log \left|\pi_{2} \circ H_{\lambda}^{+n}(x, y)\right|
$$

and by applying (2.13) inductively we obtain

$$
(1-\epsilon)^{1+d+\cdots+d^{n-1}}|y|^{d^{n}}<\left|y_{\lambda}^{n}\right|<(1+\epsilon)^{1+d+\cdots+d^{n-1}}|y|^{d^{n}} .
$$


Hence

$$
0<\log |y|+K_{1}<G_{n, \lambda}^{+}(x, y)=\frac{1}{d^{n}} \log \left|\pi_{2} \circ H_{\lambda}^{+n}(x, y)\right|<\log |y|+K_{2},
$$

with $K_{1}=\frac{d^{n}-1}{d^{n}(d-1)} \log (1-\epsilon)$ and $K_{2}=\frac{d^{n}-1}{d^{n}(d-1)} \log (1+\epsilon)$.

By (2.14) it follows that

$$
\left|G_{n+1, \lambda}^{+}(x, y)-G_{n, \lambda}^{+}(x, y)\right|=\left|d^{-n-1} \log \right| y_{\lambda}^{n+1} /\left(y_{\lambda}^{n}\right)^{d}|| \lesssim d^{-n-1}
$$

which proves that $\left\{G_{n, \lambda}^{+}\right\}$converges uniformly on $\overline{V_{R}^{+}}$. As a limit of a sequence of uniformly convergent pluriharmonic functions $\left\{G_{n, \lambda}^{+}\right\}, G_{\lambda}^{+}$is also pluriharmonic for each $\lambda \in M$ on $V_{R}^{+}$. Again by (2.14), for each $\lambda \in M$,

$$
G_{\lambda}^{+}-\log |y|
$$

is a bounded pluriharmonic function in $\overline{V_{R}^{+}}$. Therefore its restriction to vertical lines of the form $x=c$ can be continued across the point $(c, \infty)$ as a pluriharmonic function. Since

$$
\lim _{|y| \rightarrow \infty}\left(G_{\lambda}^{+}(x, y)-\log |y|\right)
$$

is bounded in $x \in \mathbb{C}$, by (2.14) it follows that $\lim _{|y| \rightarrow \infty}\left(G_{\lambda}^{+}(x, y)-\log |y|\right)$ must be a constant, say $\gamma_{\lambda}$ which also satisfies

$$
\log (1-\epsilon) /(d-1) \leq \gamma_{\lambda} \leq \log (1+\epsilon) /(d-1) .
$$

As $\epsilon>0$ is arbitrary, it follows that

$$
G_{\lambda}^{+}(x, y)=\log |y|+u_{\lambda}(x, y)
$$

on $V_{R}^{+}$where $u_{\lambda}$ is a bounded pluriharmonic function satisfying $u_{\lambda}(x, y) \rightarrow 0$ as $|y| \rightarrow \infty$.

Now fix $\lambda \in M$ and $n \geq 1$. For any $r>n$,

$$
\begin{aligned}
G_{r, \lambda}^{+}(x, y) & =d^{-r} \log ^{+}\left|H_{\lambda}^{+r}(x, y)\right| \\
& =d^{-n} G_{(r-n), \sigma^{n}(\lambda)}^{+} \circ H_{\lambda}^{+n}(x, y) .
\end{aligned}
$$

As $r \rightarrow \infty, G_{r, \lambda}^{+}$converges uniformly on $\left(H_{\lambda}^{+n}\right)^{-1}\left(V_{R}^{+}\right)$to the pluriharmonic function $d^{-n} G_{\sigma^{n}(\lambda)}^{+} \circ H_{\lambda}^{+n}$. Hence

$$
d^{n} G_{\lambda}^{+}(x, y)=G_{\sigma^{n}(\lambda)}^{+} \circ H_{\lambda}^{+n}(x, y)
$$

for $(x, y) \in\left(H_{\lambda}^{+n}\right)^{-1}\left(V_{R}^{+}\right)$. By (2.14) , for $(x, y) \in\left(H_{\lambda}^{+n}\right)^{-1}\left(V_{R}^{+}\right)$

$$
G_{r, \lambda}^{+}(x, y)=d^{-n} G_{(r-n), \sigma^{n}(\lambda)}^{+} \circ H_{\lambda}^{+n}(x, y)>d^{-n}\left(\log R+K_{1}\right)>0,
$$

for each $r>n$ which shows that

$$
G_{\lambda}^{+}(x, y) \geq d^{-n}\left(\log R+K_{1}\right)>0
$$

for $(x, y) \in\left(H_{\lambda}^{+n}\right)^{-1}\left(V_{R}^{+}\right)$. This is true for each $n \geq 1$. Hence $G_{r, \lambda}^{+}$converges uniformly to the pluriharmonic function $G_{\lambda}^{+}$on every compact set of

$$
\bigcup_{n=0}^{\infty}\left(H_{\lambda}^{+n}\right)^{-1}\left(V_{R}^{+}\right)=\mathbb{C}^{2} \backslash K_{\lambda}^{+} .
$$

Moreover, $G_{\lambda}^{+}>0$ on $\mathbb{C}^{2} \backslash K_{\lambda}^{+}$. 
Note that for each $\lambda \in M, G_{\lambda}^{+}=0$ on $K_{\lambda}^{+}$. By Remark 2.3, there exists a large enough $R>1$ so that $K_{\lambda}^{+} \subseteq V_{R} \cup V_{R}^{-}$for all $\lambda \in M$. Now choose any $A>R>1$. We will show that $\left\{G_{n, \lambda}^{+}\right\}$converges uniformly to $G_{\lambda}^{+}$on the bidisc

$$
\Delta_{A}=\{(x, y):|x| \leq A,|y| \leq A\}
$$

as $n \rightarrow \infty$. Consider the sets

$$
N=\left\{(x, y) \in \mathbb{C}^{2}:|x| \leq A\right\}, N_{\lambda}=N \cap K_{\lambda}^{+}
$$

for each $\lambda \in M$. Start with any point $z=\left(x_{0}, x_{1}\right) \in \mathbb{C}^{2}$ and define $\left(x_{i}^{\lambda}, x_{i+1}^{\lambda}\right)$ for $\lambda \in M$ and $i \geq 1$ in the following way:

$$
\left(x_{0}^{\lambda}, x_{1}^{\lambda}\right) \stackrel{H_{\lambda}^{(1)}}{\longrightarrow}\left(x_{1}^{\lambda}, x_{2}^{\lambda}\right) \stackrel{H_{\lambda}^{(2)}}{\longrightarrow} \cdots \stackrel{H_{\lambda}^{(m)}}{\longrightarrow}\left(x_{m}^{\lambda}, x_{m+1}^{\lambda}\right) \stackrel{H_{\lambda}^{(1)}}{\longrightarrow}\left(x_{m+1}^{\lambda}, x_{m+2}^{\lambda}\right) \rightarrow \cdots,
$$

where $\left(x_{0}^{\lambda}, x_{1}^{\lambda}\right)=\left(x_{0}, x_{1}\right)$ and we apply $H_{\lambda}^{(1)}, \cdots, H_{\lambda}^{(m)}$ periodically for all $\lambda \in M$. Inductively one can show that if $\left(x_{i}^{\lambda}, x_{i+1}^{\lambda}\right) \in N_{\lambda}$ for $0 \leq i \leq j-1$, then $\left|x_{i}^{\lambda}\right| \leq A$ for $0 \leq i \leq j$.

This implies that there exists $n_{0}>0$ independent of $\lambda$ so that

$$
G_{n, \lambda}^{+}(x, y)<\epsilon
$$

for all $n \geq n_{0}$ and for all $(x, y) \in N_{\lambda}$. Consider a line segment

$$
L_{a}=\{(a, w):|w| \leq A\} \subset \mathbb{C}^{2}
$$

with $|a| \leq A$. Then $G_{n, \lambda}^{+}-G_{\lambda}^{+}$is harmonic on $L_{a}^{\lambda}=\{(a, w):|w|<A\} \backslash K_{\lambda}^{+}$viewed as a subset of $\mathbb{C}$ and the boundary of $L_{a}^{\lambda}$ lies in $\{(a, w):|w|=A\} \cup\left(K_{\lambda}^{+} \cap L_{a}\right)$. By Remark 2.2, there exists $n_{1}>0$ so that

$$
-\epsilon<G_{n, \lambda}^{+}(a, w)-G_{\lambda}^{+}(a, w)<\epsilon
$$

for all $n \geq n_{1}$ and for all $(a, w) \in\{|a| \leq A,|w|=A\}$. The maximum principle shows that

$$
-\epsilon<G_{n, \lambda}^{+}(x, y)-G_{\lambda}^{+}(x, y)<\epsilon
$$

for all $n \geq \max \left\{n_{0}, n_{1}\right\}$ and for all $(x, y) \in L_{a}^{\lambda}$. This shows that for any given $\epsilon>0$, there exists $n_{2}>0$ such that

$$
-\epsilon<G_{n, \lambda}^{+}(z)-G_{\lambda}^{+}(z)<\epsilon
$$

for all $n \geq n_{2}$ and for all $(\lambda, z) \in M \times \Delta_{A}$.

Hence $G_{n, \lambda}^{+}$converges uniformly to $G_{\lambda}^{+}$on any compact subset of $\mathbb{C}^{2}$ and this is also uniform with respect to $\lambda \in M$. In particular, this implies that for each $\lambda \in M, G_{\lambda}^{+}$is continuous on $\mathbb{C}^{2}$ and pluriharmonic on $\mathbb{C}^{2} \backslash K_{\lambda}^{+}$. Moreover, $G_{\lambda}^{+}$ vanishes on $K_{\lambda}^{+}$. In particular, for each $\lambda \in M, G_{\lambda}^{+}$satisfies the submean value property on $\mathbb{C}^{2}$. Hence $G_{\lambda}^{+}$is plurisubharmonic on $\mathbb{C}^{2}$. Analogous results are true for $G_{\lambda}^{-}$.

Next, to show that the correspondence $\lambda \mapsto G_{\lambda}^{ \pm}$is continuous, take a compact set $S \subset \mathbb{C}^{2}$ and $\lambda_{0} \in M$. Then

$$
\begin{aligned}
\left|G_{\lambda}^{+}(x, y)-G_{\lambda_{0}}^{+}(x, y)\right| \leq\left|G_{n, \lambda}^{+}(x, y)-G_{\lambda}^{+}(x, y)\right|+ & \left|G_{n, \lambda}^{+}(x, y)-G_{n, \lambda_{0}}^{+}(x, y)\right| \\
& +\left|G_{n, \lambda_{0}}^{+}(x, y)-G_{\lambda_{0}}^{+}(x, y)\right|
\end{aligned}
$$

for $(x, y) \in S$. It follows from (2.17) that for given $\epsilon>0$, one can choose a large $n_{0}>0$ such that the first and third terms above are less than $\epsilon / 3$. By choosing $\lambda$ close enough to $\lambda_{0}$, it follows that $G_{n_{0}, \lambda}^{+}(x, y)$ and $G_{n_{0}, \lambda_{0}}^{+}(x, y)$ do not differ by 
more than $\epsilon / 3$. Hence the correspondence $\lambda \mapsto G_{\lambda}^{+}$is continuous. Similarly, the correspondence $\lambda \mapsto G_{\lambda}^{-}$is also continuous.

To prove that $G_{\lambda}^{+}$is Hölder continuous for each $\lambda \in M$, fix a compact $S \subset \mathbb{C}^{2}$ and let $R>1$ be such that $S$ is compactly contained in $V_{R}$. Using the continuity of $G_{\lambda}^{+}$in $\lambda$, there exists a $\delta>0$ such that $G_{\lambda}^{+}(x, y)>(d+1) \delta$ for each $\lambda \in M$ and $(x, y) \in V_{R}^{+}$. Now note that the correspondence $\lambda \mapsto K_{\lambda}^{+} \cap V_{R}$ is upper semicontinuous. Indeed, if this is not the case, then there exists a $\lambda_{0} \in M$, an $\epsilon>0$ and a sequence $\lambda_{n} \in M$ converging to $\lambda_{0}$ such that for each $n \geq 1$, there exists a point $a_{n} \in K_{\lambda_{n}}^{+} \cap V_{R}$ satisfying $\left|a_{n}-z\right| \geq \epsilon$ for all $z \in K_{\lambda_{0}}^{+}$. Let $a$ be a limit point of the $a_{n}$ 's. Then by the continuity of $\lambda \mapsto G_{\lambda}^{+}$it follows that

$$
0=G_{\lambda_{n}}^{+}\left(a_{n}\right) \rightarrow G_{\lambda}^{+}(a)
$$

which implies that $a \in K_{\lambda_{0}}^{+}$. This is a contradiction. For each $\lambda \in M$, define

$$
\Omega_{\delta}^{\lambda}=\left\{(x, y) \in V_{R}: \delta<G_{\lambda}^{+}(x, y) \leq d \delta\right\}
$$

and

$$
C_{\lambda}=\sup \left\{\left|\partial G_{\lambda}^{+} / \partial x\right|,\left|\partial G_{\lambda}^{+} / \partial y\right|:(x, y) \in \Omega_{\delta}^{\lambda}\right\} .
$$

The first observation is that the $C_{\lambda}$ 's are uniformly bounded above as $\lambda$ varies in $M$. To see this, fix $\lambda_{0} \in M$ and $\tau>0$ and let $W \subset M$ be a neighbourhood of $\lambda_{0}$ such that the sets

$$
\Omega_{W}=\overline{\bigcup_{\lambda \in W} \Omega_{\delta}^{\lambda}} \text { and } K_{W}=\overline{\bigcup_{\lambda \in W}\left(K_{\lambda}^{+} \cap V_{R}\right)}
$$

are separated by a distance of at least $\tau$. This is possible since $K_{\lambda}^{+} \cap V_{R}$ is upper semi-continuous in $\lambda$. For each $\lambda \in W, G_{\lambda}^{+}$is pluriharmonic on a fixed slightly larger open set containing $\Omega_{W}$. Cover the closure of this slightly larger open set by finitely many open balls and on each ball, the mean value property shows that the derivatives of $G_{\lambda}^{+}$are dominated by a universal constant times the sup norm of $G_{\lambda}^{+}$on it, and this in turn is dominated by the number of open balls (which is the same for all $\lambda \in W$ ) times the sup norm of $G_{\lambda}^{+}$on $V_{R}$ upto a universal constant. Since $G_{\lambda}^{+}$varies continuously in $\lambda$, it follows that the $C_{\lambda}$ 's are uniformly bounded for $\lambda \in W$ and the compactness of $M$ gives a global bound, say $C>0$ independent of $\lambda$.

Fix $\lambda_{0} \in M$ and pick $(x, y) \in S \backslash K_{\lambda_{0}}^{+}$. Let $N>0$ be such that

$$
d^{-N} \delta<G_{\lambda_{0}}^{+}(x, y) \leq d^{-N+1} \delta
$$

so that

$$
\delta<d^{N} G_{\lambda_{0}}^{+}(x, y) \leq d \delta .
$$

The assumption that $N>0$ means that $(x, y)$ is very close to $K_{\lambda_{0}}^{+}$. But

$$
d^{N} G_{\lambda_{0}}^{+}(x, y)=G_{\sigma^{N}\left(\lambda_{0}\right)}^{+} \circ H_{\lambda_{0}}^{+N}(x, y)
$$

which implies that $H_{\lambda_{0}}^{+N}(x, y) \in \Omega_{\delta}^{\sigma^{N}\left(\lambda_{0}\right)}$ where $G_{\sigma^{N}\left(\lambda_{0}\right)}^{+}$is pluriharmonic. Note that

$$
H_{\lambda_{0}}\left(V_{R} \cup V_{R}^{+}\right) \subset V_{R} \cup V_{R}^{+}, H_{\lambda_{0}}\left(V_{R}^{+}\right) \subset V_{R}^{+}
$$


which shows that $H_{\lambda_{0}}^{+k} \in V_{R}$ for all $k \leq N$ since all the $G_{\lambda}^{+}$'s are at least $(d+1) \delta$ on $V_{R}^{+}$. Differentiation of the above identity leads to

$$
\begin{aligned}
d^{N} \frac{\partial G_{\lambda_{0}}^{+}}{\partial x}(x, y)= & \frac{\partial G_{\sigma^{N}\left(\lambda_{0}\right)}^{+}}{\partial x}\left(H_{\lambda_{0}}^{+N}\right) \frac{\partial\left(\pi_{1} \circ H_{\lambda_{0}}^{+N}\right)}{\partial x}(x, y) \\
& +\frac{\partial G_{\sigma^{N}\left(\lambda_{0}\right)}^{+}}{\partial y}\left(H_{\lambda_{0}}^{+N}\right) \frac{\partial\left(\pi_{2} \circ H_{\lambda_{0}}^{+N}\right)}{\partial x}(x, y) .
\end{aligned}
$$

Let the derivatives of $H_{\lambda}$ be bounded above on $V_{R}$ by $A_{\lambda}$ and let $A=\sup A_{\lambda}<\infty$. It follows that the derivatives of $H_{\lambda_{0}}^{+N}$ are bounded above by $2^{N-1} A^{N}$ on $V_{R}$. Hence

$$
\left|d^{N} \partial G_{\lambda_{0}}^{+} / \partial x(x, y)\right| \leq C(2 A)^{N} .
$$

Let $\gamma=\log 2 A / \log d$ so that $C(2 A)^{N}=C d^{N \gamma}$. Therefore,

$$
\left|\partial G_{\lambda_{0}}^{+} / \partial x\right| \leq C d^{N(\gamma-1)} \leq C\left(d \delta / G_{\lambda_{0}}^{+}\right)^{\gamma-1}
$$

which implies that

$$
\left|\partial\left(G_{\lambda_{0}}^{+}\right)^{\gamma} / \partial x\right| \leq C \gamma(d \delta)^{\gamma-1}
$$

A similar argument can be used to bound the partial derivative of $\left(G_{\lambda_{0}}^{+}\right)^{\gamma}$ with respect to $y$. Thus the gradient of $\left(G_{\lambda_{0}}^{+}\right)^{\gamma}$ is bounded uniformly at all points that are close to $K_{\lambda_{0}}^{+}$.

Now suppose that $(x, y) \in S \backslash K_{\lambda_{0}}^{+}$is such that

$$
d^{N} \delta<G_{\lambda_{0}}^{+}(x, y) \leq d^{N+1} \delta
$$

for some $N>0$. This means that $(x, y)$ is far away from $K_{\lambda_{0}}^{+}$and the above equation can be written as

$$
\delta<d^{-N} G_{\lambda_{0}}^{+}(x, y) \leq d \delta
$$

By the surjectivity of $\sigma$, there exists a $\mu_{0} \in M$ such that $\sigma^{N}\left(\mu_{0}\right)=\lambda_{0}$. With this the invariance property of the Green functions now reads

$$
G_{\mu_{0}}^{+} \circ\left(H_{\mu_{0}}^{+N}\right)^{-1}(x, y)=d^{-N} G_{\lambda_{0}}^{+}(x, y) .
$$

The compactness of $S$ shows that there is a fixed integer $m<0$ such that if $(x, y)$ is far away from $S \backslash K_{\lambda_{0}}^{+}$, then it can be brought into the strip

$$
\left\{(x, y): \delta<G_{\lambda_{0}}^{+}(x, y) \leq d \delta\right\}
$$

by $\left(H_{\lambda}^{+|k|}\right)^{-1}$ for some $m \leq k<0$ and for all $\lambda \in M$. By enlarging $R$, we may assume that the image of $S$ under all the maps $\left(H_{\lambda}^{+|k|}\right)^{-1}, m \leq k<0$, is contained in $V_{R}$. By increasing $A$, we may also assume that all the derivatives of $H_{\lambda}$ and $H_{\lambda}^{-1}$ are bounded by $A$ on $V_{R}$. Now repeating the same argument as above, it follows that the gradient of $\left(G_{\lambda_{0}}^{+}\right)^{\gamma}$ is bounded uniformly at all points that are far away from $K_{\lambda_{0}}^{+}$- the nuance about choosing $\gamma$ as before is also valid. The choice of $\mu_{0}$ such that $\sigma^{N}\left(\mu_{0}\right)=\lambda_{0}$ is irrelevant since the derivatives involved are with respect to $x, y$ only. The only remaining case is when $(x, y) \in \Omega_{\lambda_{0}}^{\delta}$ which precisely means that $N=0$. But in this case, $\left(G_{\lambda_{0}}^{+}\right)^{\gamma-1}$ is uniformly bounded on $V_{R}$ and so are the derivatives of $G_{\lambda_{0}}^{+}$on $\Omega_{\lambda_{0}}^{\delta}$ by the reasoning given earlier. Therefore, there is a uniform bound on the gradient of $\left(G_{\lambda_{0}}^{+}\right)^{\gamma}$ everywhere on $S$. This shows that $\left(G_{\lambda_{0}}^{+}\right)^{\gamma}$ is Lipschitz on $S$ which implies that $G_{\lambda_{0}}^{+}$is Hölder continuous on $S$ with exponent 
$1 / \gamma=\log d / \log 2 A$. A set of similar arguments can be applied to deduce analogous results for $G_{\lambda}^{-}$.

\subsection{Proof of Proposition 1.2.}

Proof. We have

$$
\left(H_{\lambda}^{ \pm 1}\right)^{*}\left(\mu_{\sigma(\lambda)}^{ \pm}\right)=\left(H_{\lambda}^{ \pm 1}\right)^{*}\left(d d^{c} G_{\sigma(\lambda)}^{ \pm}\right)=d d^{c}\left(G_{\sigma(\lambda)}^{ \pm} \circ H_{\lambda}^{ \pm 1}\right)=d d^{c}\left(d G_{\lambda}^{ \pm}\right)=d \mu_{\lambda}^{ \pm}
$$

where the third equality follows from Proposition 1.1. A similar exercise shows that

$$
\left(H_{\lambda}^{ \pm 1}\right)_{*} \mu_{\lambda}^{ \pm}=d^{-1} \mu_{\sigma(\lambda)}^{ \pm} .
$$

If $\sigma$ is the identity on $M$, then

$$
G_{\lambda}^{ \pm} \circ H_{\lambda}^{ \pm 1}=d G_{\lambda}^{ \pm}
$$

which in turn imply that

$$
\left(H_{\lambda}^{ \pm 1}\right)^{*} \mu_{\lambda}=\left(H_{\lambda}^{ \pm 1}\right)^{*}\left(\mu_{\lambda}^{+} \wedge \mu_{\lambda}^{-}\right)=\left(H_{\lambda}^{ \pm 1}\right)^{*} \mu_{\lambda}^{+} \wedge\left(H_{\lambda}^{ \pm 1}\right)^{*} \mu_{\lambda}^{-}=d^{ \pm 1} \mu_{\lambda}^{+} \wedge d^{\mp 1} \mu_{\lambda}^{-}=\mu_{\lambda} .
$$

By Proposition 1.1, the support of $\mu_{\lambda}^{+}$is contained in $J_{\lambda}^{+}$. To prove the converse, let $z_{0} \in J_{\lambda}^{+}$and suppose that $\mu_{\lambda}^{+}=0$ on a neighbourhood $U_{z_{0}}$ of $z_{0}$. This means that $G_{\lambda}^{+}$is pluriharmonic on $U_{z_{0}}$ and $G_{\lambda}^{+}$attains its minimum value zero at $z_{0}$. This implies that $G_{\lambda}^{+} \equiv 0$ on $U_{z_{0}}$ which contradicts the fact that $G_{\lambda}^{+}>0$ on $\mathbb{C}^{2} \backslash K_{\lambda}^{+}$. Similar arguments can be applied to prove that $\operatorname{supp}\left(\mu_{\lambda}^{-}\right)=J_{\lambda}^{-}$.

Finally, to show that $\lambda \mapsto J_{\lambda}^{+}$is lower semi-continuous, fix $\lambda_{0} \in M$ and $\epsilon>0$. Let $x_{0} \in J_{\lambda_{0}}^{+}=\operatorname{supp}\left(\mu_{\lambda_{0}}^{+}\right)$. Then $\mu_{\lambda_{0}}^{+}\left(B\left(x_{0}, \epsilon / 2\right)\right) \neq 0$. Since the correspondence $\lambda \mapsto \mu_{\lambda}^{+}$is continuous, there exists a $\delta>0$ such that

$$
d\left(\lambda, \lambda_{0}\right)<\delta \text { implies } \mu_{\lambda}^{+}\left(B\left(x_{0} ; \epsilon / 2\right)\right) \neq 0 .
$$

Therefore, $x_{0} \in\left(J_{\lambda}^{+}\right)^{\epsilon}=\bigcup_{a \in J_{\lambda}^{+}} B(a, \epsilon)$ for all $\lambda \in M$ satisfying $d\left(\lambda, \lambda_{0}\right)<\delta$. Hence the correspondence $\lambda \mapsto J_{\lambda}^{ \pm}$is lower semi-continuous.

Let $\mathcal{L}$ be the class of plurisubharmonic functions on $\mathbb{C}^{2}$ of logarithmic growth, i.e.,

$$
\mathcal{L}=\left\{u \in \mathcal{P} \mathcal{S H}\left(\mathbb{C}^{2}\right): u(x, y) \leq \log ^{+}\|(x, y)\|+L\right\}
$$

for some $L>0$ and let

$$
\tilde{\mathcal{L}}=\left\{u \in \mathcal{P} \mathcal{S H}\left(\mathbb{C}^{2}\right): \log ^{+}\|(x, y)\|-L \leq u(x, y) \leq \log ^{+}\|(x, y)\|+L\right\}
$$

for some $L>0$. Note that there exists $L>0$ such that

$$
G_{\lambda}^{+}(z) \leq \log ^{+}\|z\|+L
$$

for all $z \in \mathbb{C}^{2}$ and for all $\lambda \in M$. Thus $G_{\lambda}^{+} \in \mathcal{L}$ for all $\lambda \in M$. For $E \subseteq \mathbb{C}^{2}$, the pluricomplex Green function of $E$ is

$$
L_{E}(z)=\sup \{u(z): u \in \mathcal{L}, u \leq 0 \text { on } E\}
$$

and let $L_{E}^{*}(z)$ be its upper semi-continuous regularization.

It turns out that the pluricomplex Green function of $K_{\lambda}^{ \pm}$is $G_{\lambda}^{ \pm}$for all $\lambda \in M$. The arguments are similar to those employed for a single Hénon map and we merely point out the salient features. Fix $\lambda \in M$. Then $G_{\lambda}^{+}=0$ on $K_{\lambda}^{+}$and $G_{\lambda}^{+} \in \mathcal{L}$. So $G_{\lambda}^{+} \leq L_{K_{\lambda}^{+}}$. To show equality, let $u \in \mathcal{L}$ be such that $u \leq 0=G_{\lambda}^{+}$on $K_{\lambda}^{+}$. By Proposition 1.1, there exists $M>0$ such that

$$
\log |y|-M<G_{\lambda}^{+}(x, y)<\log |y|+M
$$


for $(x, y) \in V_{R}^{+}$. Since $u \in \mathcal{L}$,

$$
u(x, y)-G_{\lambda}^{+}(x, y) \leq M_{1}
$$

for some $M_{1}>0$ and $(x, y) \in V_{R}^{+}$.

Fix $x_{0} \in \mathbb{C}$ and note that $u\left(x_{0}, y\right)-G_{\lambda}^{+}\left(x_{0}, y\right)$ is a bounded subharmonic function on the vertical line $T_{x_{0}}=\mathbb{C} \backslash\left(K_{\lambda}^{+} \cap\left\{x=x_{0}\right\}\right)$ and hence it can be extended across the point $y=\infty$ as a subharmonic function. Note also that

$$
u\left(x_{0}, y\right)-G_{\lambda}\left(x_{0}, y\right) \leq 0
$$

on $\partial T \subseteq K_{\lambda}^{+} \cap\left\{x=x_{0}\right\}$. By the maximum principle, it follows that $u\left(x_{0}, y\right)-$ $G_{\lambda}\left(x_{0}, y\right) \leq 0$ on $T_{x_{0}}$. This implies that $u \leq G_{\lambda}^{+}$in $\mathbb{C}^{2} \backslash K_{\lambda}^{+}$which in turn shows that $L_{K_{\lambda}^{+}}=G_{\lambda}^{+}$. Since $G_{\lambda}^{+}$is continuous on $\mathbb{C}^{2}$, we have

$$
L_{K_{\lambda}^{+}}=L_{K_{\lambda}^{+}}^{*}=G_{\lambda}^{+} .
$$

Similar arguments show that

$$
L_{K_{\lambda}^{-}}=L_{K_{\lambda}^{-}}^{*}=G_{\lambda}^{-} .
$$

Let $u_{\lambda}=\max \left\{G_{\lambda}^{+}, G_{\lambda}^{-}\right\}$. Again by Proposition 1.1, it follows that $u_{\lambda} \in \tilde{\mathcal{L}}$. For $\epsilon>0$, set $G_{\lambda, \epsilon}^{ \pm}=\max \left\{G_{\lambda}^{ \pm}, \epsilon\right\}$ and $u_{\lambda, \epsilon}=\max \left\{G_{\lambda, \epsilon}^{+}, G_{\lambda, \epsilon}^{-}\right\}$. By Bedford-Taylor,

$$
\left(d d^{c} u_{\lambda, \epsilon}\right)^{2}=d d^{c} G_{\lambda, \epsilon}^{+} \wedge d d^{c} G_{\lambda, \epsilon}^{-} .
$$

Now for a $z \in \mathbb{C}^{2} \backslash K_{\lambda}^{ \pm}$, there exists a small neighborhood $\Omega_{z} \subset \mathbb{C}^{2} \backslash K_{\lambda}^{ \pm}$of $z$ such that $\left(d d^{c} u_{\lambda, \epsilon}\right)^{2}=0$ on $\Omega_{z}$ for sufficiently small $\epsilon$. It follows that $\operatorname{supp}\left(\left(d d^{c} u_{\lambda}\right)\right)^{2} \subset$ $K_{\lambda}$.

Since $G_{\lambda}^{ \pm}=L_{K_{\lambda} \pm}^{*} \leq L_{K_{\lambda}}^{*}$, we have $u_{\lambda} \leq L_{K_{\lambda}}^{*}$. Further, note that $L_{K_{\lambda}}^{*} \leq L_{K_{\lambda}} \leq$ $0=u_{\lambda}$ almost everywhere on $K_{\lambda}$ with respect to the measure $\left(d d^{c} u_{\lambda}\right)^{2}$. This is because the set $\left\{L_{K_{\lambda}}^{*}>L_{K_{\lambda}}\right\}$ is pluripolar and consequently has measure zero with respect to $\left(d d^{c} u_{\lambda}\right)^{2}$. Therefore, $L_{K_{\lambda}}^{*} \leq u_{\lambda}$ in $\mathbb{C}^{2}$. Finally, $L_{K_{\lambda}}$ is continuous and thus $L_{K_{\lambda}}^{*}=L_{K_{\lambda}}=\max \left\{G_{\lambda}^{+}, G_{\lambda}^{-}\right\}$.

For a non-pluripolar bounded set $E$ in $\mathbb{C}^{2}$, the complex equilibrium measure is $\mu_{E}=\left(d d^{c} L_{E}^{*}\right)^{2}$. Again by Bedford-Taylor, $\mu_{K_{\lambda}}=\lim _{\epsilon \rightarrow 0}\left(d d^{c} \max \left\{G_{\lambda}^{+}, G_{\lambda}^{-}, \epsilon\right\}\right)^{2}$ which when combined with

$$
\mu_{\lambda}=\mu_{\lambda}^{+} \wedge \mu_{\lambda}^{-}=\lim _{\epsilon \rightarrow 0} d d^{c} G_{\lambda, \epsilon}^{+} \wedge d d^{c} G_{\lambda, \epsilon}^{-}
$$

and

$$
\left(d d^{c} \max \left\{G_{\lambda}^{+}, G_{\lambda}^{-}, \epsilon\right\}\right)^{2}=d d^{c} G_{\lambda, \epsilon}^{+} \wedge d d^{c} G_{\lambda, \epsilon}^{-}
$$

shows that $\mu_{\lambda}$ is the equilibrium measure of $K_{\lambda}$. Since $\operatorname{supp}\left(\mu_{\lambda}^{ \pm}\right)=J_{\lambda}^{ \pm}$, we have $\operatorname{supp}\left(\mu_{\lambda}\right) \subset J_{\lambda}$. Now note that $\mu_{\lambda}$ is the equilibrium measure of the non-pluripolar set $K_{\lambda}, L_{K_{\lambda}}^{*} \in \tilde{\mathcal{L}}$. Thus by Theorem 8.4.9 in [23], it follows that $\int_{\mathbb{C}^{2}} \mu_{\lambda}=4 \pi^{2}$ for all $\lambda \in M$.

\subsection{Proof of Theorem 1.3 .}

Proof. Let $\mathcal{L}_{y}$ be the subclass of $\mathcal{L}$ consisting of all those functions $v$ for which there exists $R>0$ such that

$$
v(x, y)-\log |y|
$$

is a bounded pluriharmonic function on $V_{R}^{+}$. 
Fix $\lambda \in M$ and let $\omega=1 / 4 d d^{c} \log \left(1+\|z\|^{2}\right)$. For a $(1,1)$ test form $\varphi$ on $\mathbb{C}^{2}$, it follows that there exists a $C>0$ such that

$$
-C\|\varphi\| \omega \leq \varphi \leq C\|\varphi\| \omega
$$

by the positivity of $\omega$.

Step 1. $S_{\lambda}$ is non-empty.

Note that

$$
\begin{aligned}
\frac{1}{d^{n}}\left|\int_{\mathbb{C}^{2}}\left(H_{\lambda}^{+n}\right)^{*}(\psi T) \wedge \varphi\right| & \lesssim \frac{\|\varphi\|}{d^{n}} \int_{\mathbb{C}^{2}}\left(H_{\lambda}^{+n}\right)^{*}(\psi T) \wedge d d^{c} \log \left(1+\|z\|^{2}\right) \\
& \lesssim \frac{\|\varphi\|}{d^{n}} \int_{\mathbb{C}^{2}} d d^{c}(\psi T) \wedge \log \left(1+\left\|\left(H_{\lambda}^{+n}\right)^{-1}(z)\right\|\right)
\end{aligned}
$$

A direct calculation shows that

$$
\frac{1}{d^{n}} \log ^{+}\left\|\left(H_{\lambda}^{+n}\right)^{-1}(z)\right\| \leq \log ^{+}|z|+C
$$

for some $C>0$, for all $n \geq 1, \lambda \in M$ and

$$
\log \left(1+\|z\|^{2}\right) \leq 2 \log ^{+}|z|+2 \log 2 .
$$

It follows that

$$
0 \leq \frac{1}{d^{n}} \log \left(1+\left\|\left(H_{\lambda}^{+n}\right)^{-1}\right\|\right) \leq 2 \log ^{+}|z|+C
$$

for some $C>0$, for all $n>0$ and $\lambda \in M$. Hence

$$
\frac{1}{d^{n}}\left|\int_{\mathbb{C}^{2}}\left(H_{\lambda}^{+n}\right)^{*}(\psi T) \wedge \varphi\right| \lesssim\|\varphi\| .
$$

The Banach-Alaoglu theorem gives that there is a subsequence $\frac{1}{d^{n_{j}^{\lambda}}}\left(H_{\lambda}^{+n_{j}^{\lambda}}\right)^{*}(\psi T)$ that converges in the sense of currents to a positive $(1,1)$-current, say $\gamma_{\lambda}$. This shows that $S_{\lambda}$ is non-empty. It also follows from the above discussion that $\int_{\mathbb{C}^{2}} \gamma_{\lambda} \wedge$ $\omega<+\infty$.

Step 2. Each $\gamma_{\lambda} \in S_{\lambda}$ is closed. Further, the support of $\gamma_{\lambda}$ is contained in $K_{\lambda}^{+}$.

Let $\chi$ be a smooth real 1 -form with compact support in $\mathbb{C}^{2}$ and let $\psi_{1} \geq 0$ be such that $\psi_{1}=1$ in a neighborhood of $\operatorname{supp}(\psi)$. Then

$$
\int_{\mathbb{C}^{2}} d \chi \wedge\left(H_{\lambda}^{+n_{j}^{\lambda}}\right)^{*}(\psi T)=\int_{\mathbb{C}^{2}} \chi \circ\left(H_{\lambda}^{+n_{j}^{\lambda}}\right)^{-1} \wedge d \psi \wedge \psi_{1} T
$$

is used to obtain the assumption that $\operatorname{supp}(\psi) \cap \operatorname{supp}(d T)=\phi$. By the CauchySchwarz inequality it follows that the term on the right above is dominated by the square root of

$$
\left(\int_{\mathbb{C}^{2}}\left((J \chi \wedge \chi) \circ\left(H_{\lambda}^{+n_{j}^{\lambda}}\right)^{-1}\right) \wedge \psi_{1} T\right)\left(\int_{\mathbb{C}^{2}} d \psi \wedge d^{c} \psi \wedge \psi_{1} T\right)
$$

whose absolute value in turn is bounded above by a harmless constant times $d^{n_{j}^{\lambda}}$. Here $J$ is the standard $\mathbb{R}$-linear map on 1 -forms satisfying $J\left(d z_{j}\right)=i d \bar{z}_{j}$ for $j=1,2$. Therefore,

$$
\mid \frac{1}{d^{n_{j}^{\lambda}}} \int_{\mathbb{C}^{2}}\left(\chi \circ\left(H_{\lambda}^{+n_{j}^{\lambda}}\right)^{-1} \wedge d \psi \wedge \psi_{1} T \mid \lesssim d^{-n_{j}^{\lambda} / 2} .\right.
$$

Evidently, the right-hand side tends to zero as $j \rightarrow \infty$. This shows that $\gamma_{\lambda}$ is closed. 
Let $R>0$ be large enough so that $\operatorname{supp}(\psi T) \cap V_{R}^{+}=\phi$. Let $z \notin K_{\lambda}^{+}$and let $B_{z}$ be a small open ball around it such that $\overline{B_{z}} \cap K_{\lambda}^{+}=\phi$. By Lemma 2.1. there exists an $N>0$ such that $H_{\lambda}^{+n}\left(B_{z}\right) \subset V_{R}^{+}$for all $n>N$. Therefore, $B_{z} \cap$ $\operatorname{supp}\left(H_{\lambda}^{+n}\right)^{*}(\psi T)=B_{z} \cap\left(H_{\lambda}^{+n}\right)^{-1}(\operatorname{supp}(\psi T))=\phi$ for all $n>N$. Since $\operatorname{supp}\left(\gamma_{\lambda}\right) \subset$ $\left.\overline{\bigcup_{n=N}^{\infty} \operatorname{supp}\left(H_{\lambda}^{+n}\right)^{*}(\psi T)}\right)$, we have $z \notin \operatorname{supp}\left(\gamma_{\lambda}\right)$. This implies $\operatorname{supp}\left(\gamma_{\lambda}\right) \subset K_{\lambda}^{+}$. Since $K_{\lambda}^{+} \cap V_{R}^{+}=\phi$ for all $\lambda \in M$, it also follows that $\operatorname{supp}\left(\gamma_{\lambda}\right)$ does not intersect $\overline{V_{R}^{+}}$.

Step 3. Each $\gamma_{\lambda}$ is a multiple of $\mu_{\lambda}^{+}$.

It follows from Proposition 8.3.6 in [23] that $\gamma_{\lambda}=c_{\gamma, \lambda} d d^{c} U_{\gamma, \lambda}$ for some $c_{\gamma, \lambda}>0$ and $U_{\gamma, \lambda} \in \mathcal{L}_{y}$. In this representation, $c_{\gamma, \lambda}$ is unique while $U_{\gamma, \lambda}$ is unique upto additive constants. We impose the following condition on $U_{\gamma, \lambda}$ :

$$
\lim _{|y| \rightarrow \infty}\left(U_{\gamma, \lambda}-\log |y|\right)=0
$$

and this uniquely determines $U_{\gamma, \lambda}$. It will suffice to show that $U_{\gamma, \lambda}=G_{\lambda}^{+}$.

Let $\gamma_{\lambda, x}$ denote the restriction of $\gamma_{\lambda}$ to the plane $\{(x, y): y \in \mathbb{C}\}$. Since $U_{\gamma, \lambda} \in$ $\mathcal{L}_{y}$, it follows that

$$
\int_{\mathbb{C}} \gamma_{\lambda, x}=2 \pi c_{\gamma, \lambda}, \quad U_{\gamma, \lambda}(x, y)=\frac{1}{2 \pi c_{\gamma, \lambda}} \int_{\mathbb{C}} \log |y-\zeta| \gamma_{\lambda, x}(\zeta) .
$$

Consider a uniform filtration $V_{R}^{ \pm}, V_{R}$ for all the maps $H_{\lambda}$ where $R^{d}>2 R$ and $\left|p_{j, \lambda}(y)\right| \geq|y|^{d} / 2$ for $|y| \geq R$. Let $0 \neq a=\sup \left|a_{j}(\lambda)\right|<\infty$ (where the supremum is taken over all $1 \leq j \leq m$ and $\lambda \in M)$ and choose $R_{1}>R^{d} / 2$. Define

$$
A=\left\{(x, y) \in \mathbb{C}^{2}:|y|^{d} \geq 2(1+a)|x|+2 R_{1}\right\} .
$$

Evidently, $A \subset\{|y|>R\}$. Lemma 2.1 shows that for all $\lambda \in M, H_{\lambda}(x, y) \subset V_{R}^{+}$ when $(x, y) \in A \cap V_{R}^{+}$. Furthermore, for $(x, y) \in A \cap\left(\mathbb{C}^{2} \backslash V_{R}^{+}\right)$, it follows that

$$
\left|p_{j, \lambda}(y)-a_{j}(\lambda) x\right| \geq|y|^{d} / 2-a|x| \geq|y|+R \text {. }
$$

This shows that $H_{\lambda}(A) \subset V_{R}^{+}$. By Lemma 2.1, again it can be seen that $H_{\lambda}^{+n}(A) \subset$ $V_{R}^{+}$for all $n \geq 1$ which shows that $A \cap K_{\lambda}^{+}=\phi$ for all $\lambda \in M$. Let $C>0$ be such that

$$
C^{d} \geq \max \left\{2(1+|a|), 2 R_{1}\right\} .
$$

If $|y| \geq C\left(|x|^{1 / d}+1\right)$, then

$$
|y|^{d} \geq C^{d}(|x|+1) \geq 2(1+|a|)|x|+2 R_{1}
$$

which implies that

$$
B=\left\{(x, y) \in \mathbb{C}^{2}:|y| \geq C\left(|x|^{1 / d}+1\right)\right\} \subset A
$$

and hence $K_{\lambda}^{+} \cap B=\phi$. Since $V_{R}^{+} \subset B$ for sufficiently large $R$, by applying Lemma 2.1 once again it follows that

$$
K_{\lambda}^{+} \cap B=\phi \text { and } \bigcup_{n=0}^{\infty}\left(H_{\lambda}^{+n}\right)^{-1}(B)=\mathbb{C}^{2} \backslash K_{\lambda}^{+}
$$

for all $\lambda \in M$.

Set $r=C\left(|x|^{1 / d}+1\right)$. Since $\operatorname{supp}\left(\gamma_{\lambda}\right) \subset K_{\lambda}^{+}$, it follows that

$$
\operatorname{supp}\left(\gamma_{\lambda, x}\right) \subset\{|y| \leq r\}
$$


for all $\lambda \in M$. Since

$$
|y|-r \leq|y-\zeta| \leq|y|+r
$$

for $|y|>r$ and $|\zeta| \leq r$, (2.18) yields

$$
\log (|y|-r) \leq U_{\gamma, \lambda}(x, y) \leq \log (|y|+r)
$$

which implies that

$$
-(r /|y|) /(1-r /|y|) \leq U_{\gamma, \lambda}(x, y)-\log |y| \leq r /|y| .
$$

Hence for $|y|>2 r$, we get

$$
-2 r /|y| \leq U_{\gamma, \lambda}(x, y)-\log |y| \leq r /|y|
$$

for all $\lambda \in M$.

For each $N \geq 1$, let $\gamma_{\lambda}(N)=d^{N}\left(H_{\lambda}^{+N}\right)_{*}\left(\gamma_{\lambda}\right)$. Then

$$
\gamma_{\lambda}(N)=\lim _{j \rightarrow \infty} d^{-n_{j}+N}\left(H_{\sigma^{N}(\lambda)}^{+\left(n_{j}-N\right)}\right)^{*}(\psi T) \in S_{\sigma^{N}(\lambda)}(\psi T) .
$$

Therefore,

$$
\gamma_{\sigma^{N}(\lambda)}=c_{\gamma, \sigma^{N}(\lambda)} d d^{c} U_{\gamma, \sigma^{N}(\lambda)}
$$

for some $c_{\gamma, \sigma^{N}(\lambda)}>0$ and $U_{\gamma, \sigma^{N}(\lambda)} \in \mathcal{L}_{y}$ and, moreover,

$$
c_{\gamma, \lambda} d d^{c} U_{\gamma, \lambda}=\gamma_{\lambda}=d^{-N}\left(H_{\lambda}^{+N}\right)^{*} \gamma_{\sigma^{N}(\lambda)}=c_{\gamma, \sigma^{N}(\lambda)} d d^{c}\left(d^{-N}\left(H_{\lambda}^{+N}\right)^{*} U_{\gamma, \sigma^{N}(\lambda)}\right) .
$$

Note that both $d^{-N}\left(H_{\lambda}^{+N}\right)^{*} U_{\gamma, \sigma^{N}(\lambda)}$ and $U_{\gamma, \sigma^{N}(\lambda)}$ belong to $\mathcal{L}_{y}$. It follows that $c_{\gamma, \lambda}=c_{\gamma, \sigma^{N}(\lambda)}$ and $d^{-N}\left(H_{\lambda}^{+N}\right)^{*} U_{\gamma, \sigma^{N}(\lambda)}$ and $U_{\gamma, \lambda}$ coincide up to an additive constant which can be shown to be zero as follows.

By the definition of the class $\mathcal{L}_{y}$, there exists a pluriharmonic function $u_{\lambda, N}$ on some $V_{R}^{+}$such that

$$
U_{\gamma, \sigma^{N}(\lambda)}(x, y)-\log |y|=u_{\lambda, N} \text { and } \lim _{|y| \rightarrow \infty} u_{\lambda, N}(x, y)=u_{0} \in \mathbb{C} .
$$

Therefore, if $(x, y) \in\left(H_{\lambda}^{+N}\right)^{-1}\left(V_{R}^{+}\right)$and $\left(x_{N}^{\lambda}, y_{N}^{\lambda}\right)=H_{\lambda}^{+N}(x, y)$, then

$$
d^{-N}\left(H_{\lambda}^{+N}\right)^{*} U_{\gamma, \sigma^{N}(\lambda)}(x, y)-d^{-N} \log \left|y_{N}^{\lambda}\right|=d^{-N} u_{\lambda, N}\left(x_{N}^{\lambda}, y_{N}^{\lambda}\right) .
$$

By (2.15), we have that

$$
d^{-N} \log \left|y_{N}^{\lambda}\right|-\log |y| \rightarrow 0
$$

as $|y| \rightarrow \infty$ which shows that

$$
d^{-N}\left(H_{\lambda}^{+N}\right)^{*} U_{\gamma, \sigma^{N}(\lambda)}(x, y)-\log |y| \rightarrow 0
$$

as $|y| \rightarrow \infty$. But by definition,

$$
U_{\gamma, \lambda}(x, y)-\log |y| \rightarrow 0
$$

as $|y| \rightarrow \infty$ and this shows that $d^{-N}\left(H_{\lambda}^{+N}\right)^{*} U_{\gamma, \sigma^{N}(\lambda)}=U_{\gamma, \lambda}$.

Let $(x, y) \in \mathbb{C}^{2} \backslash K_{\lambda}^{+}$and $\epsilon>0$. For a sufficiently large $n,\left(x_{n}^{\lambda}, y_{n}^{\lambda}\right)=H_{\lambda}^{+n}(x, y)$ satisfies $\left|x_{n}^{\lambda}\right| \leq\left|y_{n}^{\lambda}\right|$ and $\left(x_{n}^{\lambda}, y_{n}^{\lambda}\right) \in B$ as defined above. Hence by (2.23), we get

$$
\left|d^{-n}\left(H_{\lambda}^{+n}\right)^{*} U_{\gamma, \sigma^{n}(\lambda)}-d^{-n} \log \right| y_{n}^{\lambda}|| \leq \frac{2 C}{d^{n}\left|y_{n}^{\lambda}\right|}\left(\left|x_{n}^{\lambda}\right|^{1 / d}+1\right)<\epsilon .
$$

On the other hand, by using (2.15), it follows that

$$
\left|G_{\lambda}^{+}(x, y)-d^{-n} \log \right| y_{n}^{\lambda}||<\epsilon
$$


for large $n$. Combining these two inequalities and the fact that $d^{-n}\left(H_{\lambda}^{+n}\right)^{*} U_{\gamma, \sigma^{n}(\lambda)}$ $=U_{\gamma, \lambda}$ for all $n \geq 1$, we get

$$
\left|G_{\lambda}^{+}(z)-U_{\gamma, \lambda}(z)\right|<2 \epsilon .
$$

Hence $U_{\gamma, \lambda}=G_{\lambda}^{+}$in $\mathbb{C}^{2} \backslash K_{\lambda}^{+}$.

The next step is to show that $U_{\gamma, \lambda}=0$ in the interior of $K_{\lambda}^{+}$. Since $U_{\gamma, \lambda}=G_{\lambda}^{+}$ in $\mathbb{C}^{2} \backslash K_{\lambda}^{+}$, the maximum principle applied to $U_{\gamma, \lambda}(x,$.$) with x$ being fixed, gives $U_{\gamma, \lambda} \leq 0$ on $K_{\lambda}^{+}$. Suppose that there exists a non-empty $\Omega \subset \subset K_{\lambda}^{+}$satisfying $U_{\gamma, \lambda} \leq-t$ in $\Omega$ with $t>0$. Let $R>0$ be so large that $\bigcup_{n=0}^{\infty} H_{\lambda}^{+n}(\Omega) \subset V_{R}-$ this follows from Lemma 2.1. Since $d^{-n}\left(H_{\lambda}^{+n}\right)^{*} U_{\gamma, \sigma^{n}(\lambda)}=U_{\gamma, \lambda}$ for each $n \geq 1$, it follows that

$$
H_{\lambda}^{+n}(\Omega) \subset\left\{U_{\gamma, \sigma^{n}(\lambda)} \leq-d^{n} t\right\} \cap V_{R}
$$

for each $n \geq 1$. The measure of the last set with $x$ fixed and $|x| \leq R$ can be estimated in this way: let

$$
Y_{x}=\left\{y \in \mathbb{C}: U_{\gamma, \sigma^{n}(\lambda)} \leq-d^{n} t\right\} \cap\{|y|<R\} .
$$

By the definition of capacity

$$
\operatorname{cap}\left(Y_{x}\right) \leq \exp \left(-d^{n} t\right)
$$

and since the Lebesgue measure of $Y_{x}$, say $m\left(Y_{x}\right)$ is at most $\pi e c a p\left(Y_{x}\right)^{2}$ (by the compactness of $Y_{x} \subset \mathbb{C}$ ), we get

$$
m\left(Y_{x}\right) \leq \pi \exp \left(1-2 d^{n} t\right)
$$

Now for each $\lambda \in M$, the Jacobian determinant of $H_{\lambda}$ is a constant given by $a_{\lambda}=a_{1}(\lambda) a_{2}(\lambda) \cdots a_{m}(\lambda) \neq 0$ and since the correspondence $\lambda \mapsto a_{\lambda}$ is continuous, an application of Fubini's theorem yields

$$
\begin{aligned}
a^{n} m(\Omega) & \leq\left|a_{\sigma^{n-1}(\lambda)} \cdots a_{\lambda}\right| m(\Omega)=m\left(H_{\lambda}^{+n}(\Omega)\right) \leq \int_{|x| \leq R} m\left(Y_{x}\right) d v_{x} \\
& \leq \pi^{2} R^{2} \exp \left(1-2 d^{n} t\right)
\end{aligned}
$$

where $a=\inf _{\lambda \in M}\left|a_{\lambda}\right|$. This is evidently a contradiction for large $n$ if $m(\Omega)>0$.

So far, it has been shown that $U_{\gamma, \lambda}=G_{\lambda}^{+}$in $\mathbb{C}^{2} \backslash J_{\lambda}^{+}$. By using the continuity of $G_{\lambda}^{+}$and the upper semi-continuity of $U_{\gamma, \lambda}$, we have that $U_{\gamma, \lambda} \geq G_{\lambda}^{+}$in $\mathbb{C}^{2}$. Let $\epsilon>0$ and consider the slice $D_{\lambda}=\left\{y: G_{\lambda}^{+}<\epsilon\right\}$ in the $y$-plane for some fixed $x$. Note that $U_{\gamma, \lambda}(x,)=.G_{\lambda}^{+}(x,)=.\epsilon$ on the boundary $\partial D$. Hence by the maximum principle, $U_{\gamma, \lambda}(x,.) \leq \epsilon$ in $D_{\lambda}$. Since $x$ and $\epsilon$ are arbitrary, it follows that $U_{\gamma, \lambda}=G_{\lambda}^{+}$in $\mathbb{C}^{2}$. This implies that

for any $\gamma_{\lambda} \in S_{\lambda}(\psi T)$.

$$
\gamma_{\lambda}=c_{\gamma, \lambda} \mu_{\lambda}^{+}
$$

This completes the proof of Theorem 1.3 .

\subsection{Proof of Proposition 1.4.}

Proof. Let $\sigma: M \rightarrow M$ be an arbitrary continuous map and pick a $\gamma_{\lambda} \in S(\psi, T)$. Let $\theta=1 / 2 d d^{c} \log \left(1+|x|^{2}\right)$ in $\mathbb{C}^{2}$ (with coordinates $x, y$ ) which is a positive closed $(1,1)$-current depending only on $x$. Then for any test function $\varphi$ on $\mathbb{C}^{2}$,

$$
\int_{\mathbb{C}^{2}} \varphi \gamma_{\lambda} \wedge \theta=c_{\gamma, \lambda} \int_{\mathbb{C}^{2}} U_{\gamma, \lambda} d d^{c} \varphi \wedge \theta=c_{\gamma, \lambda} \int_{\mathbb{C}} \theta \int_{\mathbb{C}} U_{\gamma, \lambda} \Delta_{y} \varphi=c_{\gamma, \lambda} \int_{\mathbb{C}} \theta \int_{\mathbb{C}} \varphi \Delta_{y} U_{\gamma, \lambda} .
$$


Since $y \mapsto U_{\gamma, \lambda}(x, y)$ has logarithmic growth near infinity and $\varphi$ is arbitrary, it follows that

$$
\int_{\mathbb{C}^{2}} \gamma_{\lambda} \wedge \theta=2 \pi c_{\gamma, \lambda} \int_{\mathbb{C}^{2}} \theta=(2 \pi)^{2} c_{\gamma, \lambda}
$$

Let $R>0$ be large enough so that $\operatorname{supp}(\psi T) \cap V_{R}^{+}=\phi$, which implies that $\operatorname{supp}(\psi T)$ is contained in the closure of $V_{R} \cup V_{R}^{-}$. Then

$$
\begin{aligned}
\int_{\mathbb{C}^{2}} \frac{1}{d^{n_{j}^{\lambda}}}\left(H_{\lambda}^{+n_{j}^{\lambda}}\right)^{*}(\psi T) \wedge \theta & =\frac{1}{d^{n_{j}^{\lambda}}} \int_{\mathbb{C}^{2}} \psi T \wedge \frac{1}{2}\left(H_{\lambda}^{+n_{j}^{\lambda}}\right)_{*} d d^{c} \log \left(1+|x|^{2}\right) \\
& =\frac{1}{d^{n_{j}^{\lambda}}} \int_{\mathbb{C}^{2}}(\psi T) \wedge d d^{c}\left(\frac{1}{2} \log \left(1+\left|\pi_{1} \circ\left(H_{\lambda}^{+n_{j}^{\lambda}}\right)^{-1}\right|^{2}\right)\right) \\
& =\frac{1}{d^{n_{j}^{\lambda}}} \int_{\frac{V_{R} \cup V_{R}^{-}}{V^{2}}} \psi \wedge d d^{c}\left(\frac{1}{2} \log \left(1+\left|\pi_{1} \circ\left(H_{\lambda}^{+n_{j}^{\lambda}}\right)^{-1}\right|^{2}\right)\right) .
\end{aligned}
$$

It is therefore sufficient to study the behavior of $\log \left(1+\left|\pi_{1} \circ\left(H_{\lambda}^{+n_{j}^{\lambda}}\right)^{-1}\right|^{2}\right)$. But

$$
\log ^{+}|x| \leq \log ^{+}|(x, y)| \leq \log ^{+}|x|+R
$$

for $(x, y) \in V_{R} \cup V_{R}^{-}$and by combining this with

$$
2 \log ^{+}|x| \leq \log \left(1+|x|^{2}\right) \leq 2 \log ^{+}|x|+\log 2,
$$

it follows that the behavior of $(1 / 2) d^{-n_{j}^{\lambda}} \log \left(1+\left|\pi_{1} \circ\left(H_{\lambda}^{+n_{j}^{\lambda}}\right)^{-1}\right|^{2}\right)$ as $j \rightarrow \infty$ is similar to that of $d^{-n_{j}^{\lambda}} \log ^{+}\left|\left(H_{\lambda}^{+n_{j}^{\lambda}}\right)^{-1}\right|$.

Now suppose that $\sigma$ is the identity on $M$. In this case, $\left(H_{\lambda}^{+n_{j}^{\lambda}}\right)^{-1}$ is just the usual $n_{j}^{\lambda}$-fold iterate of the map $H_{\lambda}$ and by Proposition 1.1] it follows that

$$
\lim _{j \rightarrow \infty} d^{-n_{j}^{\lambda}} \log \left\|\left(H_{\lambda}^{+n_{j}^{\lambda}}\right)^{-1}\right\|=G_{\lambda}^{-}
$$

and hence

$$
4 \pi^{2} c_{\gamma, \lambda}=\int_{\mathbb{C}^{2}} \gamma_{\lambda} \wedge \theta=\int_{\mathbb{C}^{2}} \lim _{j \rightarrow \infty} \frac{1}{d^{n_{j}^{\lambda}}}\left(H_{\lambda}^{+n_{j}^{\lambda}}\right)^{*}(\psi T) \wedge \theta=\int_{\mathbb{C}^{2}} \psi T \wedge \mu_{\lambda}^{-} .
$$

The right-hand side of the above equation is independent of the subsequence used in the construction of $\gamma_{\lambda}$ and hence $S_{\lambda}(\psi, T)$ contains a unique element.

The other case to consider is when there exists a $\lambda_{0} \in M$ such that $\sigma^{n}(\lambda) \rightarrow \lambda_{0}$ for all $\lambda$. For each $n \geq 1$, let

$$
\tilde{G}_{n, \lambda}^{-}=\frac{1}{d^{n}} \log ^{+}\left\|\left(H_{\lambda}^{+n}\right)^{-1}\right\| .
$$

Note that $\tilde{G}_{n, \lambda}^{-} \neq G_{n, \lambda}^{-}$! It will suffice to show that $\tilde{G}_{n, \lambda}^{-}$converges uniformly on compact subsets of $\mathbb{C}^{2}$ to a plurisubharmonic function, say $\tilde{G}_{\lambda}^{-}$. Let

$$
\tilde{K}_{\lambda}^{-}=\left\{z \in \mathbb{C}^{2}: \text { the sequence }\left\{\left(H_{\lambda}^{+n}\right)^{-1}(z)\right\} \text { is bounded }\right\}
$$

and let $A \subset \mathbb{C}^{2}$ be a relatively compact set such that $A \cap \tilde{K}_{\lambda}^{-}=\phi$ for all $\lambda \in M$. The arguments used in Lemma 2.1 show that

$$
\mathbb{C}^{2} \backslash \tilde{K}_{\lambda}^{-}=\bigcup_{n=0}^{\infty} H_{\lambda}^{+n}\left(V_{R}^{-}\right)
$$


for a sufficiently large $R>0$. As Proposition [1.1] it can be shown that $\tilde{G}_{n, \lambda}^{-}$ converges to a pluriharmonic function $\tilde{G}_{\lambda}^{-}$on $V_{R}^{-}$. Hence for large $m, n$,

$$
\left|\tilde{G}_{m, \lambda}^{-}(p)-\tilde{G}_{n, \lambda}^{-}(q)\right|<\epsilon
$$

for $p, q \in V_{R}^{-}$that are close enough. Let $n_{0}$ be such that $\left(H_{\lambda_{0}}^{+n_{0}}\right)^{-1}(A) \subset V_{R}^{-}$and pick a relatively compact set $S \subset V_{R}^{-}$such that $\left(H_{\lambda_{0}}^{+n_{0}}\right)^{-1}(A) \subset S$. Pick any $\lambda$. Since $\sigma^{n}(\lambda) \rightarrow \lambda_{0}$ and the maps $H_{\lambda}^{ \pm 1}$ depend continuously on $\lambda$, it follows that $H_{\sigma^{n}(\lambda)}^{+n_{0}}(A) \subset S$. By choosing $m, n$ large enough, it is possible to ensure that for all $(x, y) \in A,\left(H_{\sigma^{m-n_{0}(\lambda)}}^{+n_{0}}\right)^{-1}(x, y)$ and $\left(H_{\sigma^{n-n_{0}(\lambda)}}^{+n_{0}}\right)^{-1}(x, y)$ are as close to each other as desired. By writing

$$
\tilde{G}_{n, \lambda}^{-}(x, y)=\frac{1}{d^{n_{0}}} \frac{1}{d^{n-n_{0}}} \log ^{+}\left\|H_{\lambda}^{-1} \circ \cdots \circ H_{\sigma^{n-n_{0}+1}(\lambda)}^{-1} \circ\left(H_{\sigma^{n-n_{0}}(\lambda)}^{+n_{0}}\right)^{-1}(x, y)\right\|
$$

and using (2.25) it follows that $\tilde{G}_{n, \lambda}^{-}$converges uniformly to a pluriharmonic function on $A$. To conclude that this convergence is actually uniform on compact sets of $\mathbb{C}^{2}$, it suffices to appeal to the arguments used in Proposition 1.1.

\subsection{Proof of Theorem 1.5.}

Proof. Recall that now $\sigma$ is the identity and

$$
H(\lambda, x, y)=\left(\lambda, H_{\lambda}(x, y)\right)
$$

Thus the second coordinate of the $n$-fold iterate of $H$ is simply the $n$-fold iterate $H_{\lambda} \circ H_{\lambda} \circ \cdots \circ H_{\lambda}(x, y)$. For simplicity, this will be denoted by $H_{\lambda}^{n}$ as opposed to $H_{\lambda}^{+n}$ since they both represent the same map. Consider the $\operatorname{disc} \mathcal{D}=\{x=0,|y|<$ $R\} \subset \mathbb{C}^{2}$ and let $0 \leq \psi \leq 1$ be a test function with compact support in $\mathcal{D}$ such that $\psi \equiv 1$ in a $\mathcal{D}_{r}=\{x=0,|y|<r\}$ where $r<R$. Let $\imath: \mathcal{D} \rightarrow V_{R}$ be the inclusion map. Let $L$ be a smooth subharmonic function of $|y|$ on the $y$-plane such that $L(y)=\log |y|$ for $|y|>R$ and define $\Theta=(1 / 2 \pi) d d^{c} L$. If $\pi_{y}$ is the projection from $\mathbb{C}^{2}$ onto the $y$-axis, let

$$
\alpha_{n, \lambda}=\left.\left(\pi_{y} \circ H_{\lambda}^{n} \circ \imath\right)^{*} \Theta\right|_{\mathcal{D}_{r}} .
$$

By using Theorem 1.3 and Proposition 1.4 along with Lemma 4.1 in [3] it follows that if $j_{n}$ is a sequence such that $1 \leq j_{n}<n$ and both $j_{n}, n-j_{n} \rightarrow \infty$, then

$$
\lim _{n \rightarrow \infty} d^{-n}\left(H_{\lambda}^{j_{n}}\right)_{*} \alpha_{n, \lambda}=\frac{c_{\lambda} \mu_{\lambda}}{2 \pi}
$$

where $c_{\lambda}=\frac{1}{4 \pi^{2}} \int \psi[\mathcal{D}] \wedge \mu_{\lambda}^{+}$. Note that $c_{\lambda}=1 / 2 \pi$ for all $\lambda \in M$ since $\mu_{\lambda}^{+}=d d^{c} G_{\lambda}^{+}$ and $G_{\lambda}^{+}=\log |y|$ plus a harmonic term in $V_{R}^{+}$. As a consequence, if $\sigma_{n, \lambda}=d^{-n} \alpha_{n, \lambda}$ and

$$
\mu_{n, \lambda}=\frac{1}{n} \sum_{j=0}^{n-1}\left(H_{\lambda}^{j}\right)_{*}\left(\sigma_{n, \lambda}\right),
$$

then Lemma 4.2 in [3] shows that

$$
\lim _{n \rightarrow \infty} \mu_{n, \lambda}=\frac{\mu_{\lambda}}{4 \pi^{2}}
$$

for each $\lambda \in M$. 
For an arbitrary compactly supported probability measure $\mu^{\prime}$ on $M$ and for each $n \geq 0$ let $\mu_{n}$ and $\sigma_{n}$ be defined by the recipe in (1.2), i.e., for a test function $\varphi$,

$$
\left\langle\mu_{n}, \varphi\right\rangle=\int_{M}\left(\int_{\{\lambda\} \times \mathbb{C}^{2}} \varphi \mu_{n, \lambda}\right) \mu^{\prime}(\lambda) \text { and }\left\langle\sigma_{n}, \varphi\right\rangle=\int_{M}\left(\int_{\{\lambda\} \times \mathbb{C}^{2}} \varphi \sigma_{n, \lambda}\right) \mu^{\prime}(\lambda) \text {. }
$$

We claim that

$$
\lim _{n \rightarrow \infty} \mu_{n}=\mu \text { and } \mu_{n}=\frac{1}{n} \sum_{j=0}^{n-1} H_{*}^{j} \sigma_{n}
$$

where $H$ is as in (2.26). For the first claim, note that for all test functions $\varphi$,

$$
\begin{aligned}
\lim _{n \rightarrow \infty}\left\langle\mu_{n}, \varphi\right\rangle & =\lim _{n \rightarrow \infty} \int_{M}\left\langle\mu_{n, \lambda}, \varphi\right\rangle \mu^{\prime}(\lambda)=\int_{M} \lim _{n \rightarrow \infty}\left\langle\mu_{n, \lambda}, \varphi\right\rangle \mu^{\prime}(\lambda) \\
& =\int_{M}\left\langle\frac{\mu_{\lambda}}{4 \pi^{2}}, \varphi\right\rangle \mu^{\prime}(\lambda)=\langle\mu, \varphi\rangle
\end{aligned}
$$

where the second equality follows by the dominated convergence theorem. For the second claim, note that

$$
\left\langle\frac{1}{n} \sum_{j-0}^{n-1} H_{*}^{j} \sigma_{n}, \varphi\right\rangle=\int_{M}\left\langle\frac{1}{n} \sum_{j=0}^{n-1} H_{\lambda *}^{j}\left(\sigma_{n, \lambda}\right), \varphi\right\rangle \mu^{\prime}(\lambda)=\int_{M}\left\langle\mu_{n, \lambda}, \varphi\right\rangle \mu^{\prime}(\lambda)=\left\langle\mu_{n}, \varphi\right\rangle .
$$

Hence by (2.28), we get

$$
\lim _{n \rightarrow \infty} \frac{1}{n} \sum_{j-0}^{n-1} H_{*}^{j} \sigma_{n}=\mu .
$$

Note that the support of $\mu$ is contained in $\operatorname{supp}\left(\mu^{\prime}\right) \times V_{R}$. Let $\mathcal{P}$ be a partition of $M \times V_{R}$ so that the $\mu$-measure of the boundary of each element of $\mathcal{P}$ is zero and each of its elements has diameter less than $\epsilon$. This choice is possible by Lemma 8.5 in [25]. For each $n \geq 0$, define the $d_{n}$ metric on $M \times V_{R}$ by

$$
d_{n}(p, q)=\max _{0 \leq i \leq n-1} d\left(H^{i}(p), H^{i}(q)\right)
$$

where $d$ is the product metric on $M \times V_{R}$. Note that each element $\mathcal{B}$ of $\bigvee_{j=0}^{n-1} H^{-j} \mathcal{P}$ is inside an $\epsilon$-ball in the $d_{n}$ metric and if $\mathcal{B}_{\lambda}=(\mathcal{B} \times\{\lambda\}) \cap V_{R}$, then the $\sigma_{n}$ measure of $\mathcal{B}$ is given by

$$
\begin{aligned}
\sigma_{n}(\mathcal{B})=\int_{M} \sigma_{n, \lambda}\left(\mathcal{B}_{\lambda}\right) \mu^{\prime}(\lambda) & =\int_{M}\left(d^{-n} \int_{\mathcal{B}_{\lambda} \cap \mathcal{D}} H_{\lambda}^{n *} \Theta\right) \mu^{\prime}(\lambda) \\
& =\int_{M}\left(d^{-n} \int_{H_{\lambda}^{n}\left(\mathcal{B}_{\lambda} \cap \mathcal{D}\right)} \Theta\right) \mu^{\prime}(\lambda) .
\end{aligned}
$$

Therefore, since $\Theta$ is bounded above on $\mathbb{C}^{2}$, there exists $C>0$ such that (2.29)

$$
\sigma_{n}(\mathcal{B}) \leq C d^{-n} \int_{M} \operatorname{Area}\left(H_{\lambda}^{n}\left(\mathcal{B}_{\lambda} \cap \mathcal{D}\right)\right) \mu^{\prime}(\lambda)=C d^{-n} \text { Area }\left(H^{n}(\mathcal{B} \cap(\mathcal{D} \times M))\right) .
$$


For a continuous map $f: X \rightarrow X$ on a compact set $X$ endowed with an invariant probability measure $m$, let

$$
\begin{aligned}
\mathcal{H}_{m}(\mathcal{A}) & =-\sum_{i=1}^{k} m\left(A_{i}\right) \log m\left(A_{i}\right), \\
h(\mathcal{A}, f) & =\lim _{n \rightarrow \infty} \frac{1}{n} \mathcal{H}_{m}\left(\bigvee_{j=0}^{n-1} f^{-j} \mathcal{A}\right)
\end{aligned}
$$

for a partition $\mathcal{A}=\left\{A_{1}, A_{2}, \ldots, A_{k}\right\}$ of $X$. By definition, the measure theoretic entropy of $f$ with respect to $m$ is $h_{m}(f)=\sup _{\mathcal{A}} h(\mathcal{A}, f)$. We will work with $X=\operatorname{supp}(\mu) \subset M \times V_{R}$ and view $H$ as a self map of $X$.

If $v^{0}(H, n, \epsilon)$ denotes the supremum of the areas of $H^{n}(\mathcal{B} \cap(\mathcal{D} \times M))$ over all $\epsilon$-balls $\mathcal{B}$, then

$$
\mathcal{H}_{\sigma_{n}}\left(\bigvee_{j=0}^{n-1} H^{-j} \mathcal{P}\right) \geq-\log C+n \log d-\log v^{0}(H, n, \epsilon)
$$

by (2.29). By appealing to Misiurewicz's variational principle as explained in 3. we get a lower bound for the measure theoretic entropy $h_{\mu}$ of $H$ with respect to the invariant probability measure $\mu$ as follows:

$$
h_{\mu} \geq \limsup _{n \rightarrow \infty} \frac{1}{n}\left(-\log C+n \log d-\log v^{0}(H, n, \epsilon)\right) \geq \log d-\limsup _{n \rightarrow \infty} v^{0}(H, n, \epsilon) .
$$

By Yomdin's result ([24]), it follows that $\lim _{\epsilon \rightarrow 0} v^{0}(H, n, \epsilon)=0$. Thus $h_{\mu} \geq \log d$. To conclude, note that $\operatorname{supp}(\mu) \subset \mathcal{J} \subset M \times V_{R}$ and therefore by the variational principle the topological entropy of $H$ on $\mathcal{J}$ is also at least $\log d$.

\section{Fibered FAMILIES OF HOLOMORPHIC ENDOMORPHISM OF $\mathbb{P}^{k}$}

\subsection{Proof of Proposition 1.6.}

Proof. By (1.4), there exists a $C>1$ such that

$C^{-1}\left\|F_{\sigma^{n-1}(\lambda)} \circ \cdots \circ F_{\lambda}(x)\right\|^{d} \leq\left\|F_{\sigma^{n}(\lambda)} \circ \cdots \circ F_{\lambda}(x)\right\| \leq C\left\|F_{\sigma^{n-1}(\lambda)} \circ \cdots \circ F_{\lambda}(x)\right\|^{d}$

for all $\lambda \in M, x \in \mathbb{C}^{k+1}$ and for all $n \geq 1$. Consequently,

$$
\left|G_{n+1, \lambda}(x)-G_{n, \lambda}(x)\right| \leq \log C / d^{n+1} .
$$

Hence for each $\lambda \in M$, as $n \rightarrow \infty, G_{n, \lambda}$ converges uniformly to a continuous plurisubharmonic function $G_{\lambda}$ on $\mathbb{C}^{k+1}$. If $G_{n}(\lambda, x)=G_{n, \lambda}(x)$, then (3.1) shows that $G_{n} \rightarrow G$ uniformly on $M \times\left(\mathbb{C}^{k+1} \backslash\{0\}\right)$.

Furthermore, for $\lambda \in M$ and $c \in \mathbb{C}^{*}$,

$$
\begin{aligned}
G_{\lambda}(c x) & =\lim _{n \rightarrow \infty} \frac{1}{d^{n}} \log \left\|F_{\sigma^{n-1}(\lambda)} \circ \cdots \circ F_{\lambda}(c x)\right\| \\
& =\lim _{n \rightarrow \infty}\left(\frac{1}{d^{n}} \log |c|^{d^{n}}+\frac{1}{d^{n}} \log \left\|F_{\sigma^{n-1}(\lambda)} \circ \cdots \circ F_{\lambda}(z)\right\|\right)=\log |c|+G_{\lambda}(x) .
\end{aligned}
$$

We also note that

$$
G_{\sigma(\lambda)} \circ F_{\lambda}(x)=d \lim _{n \rightarrow \infty} \frac{1}{d^{n+1}} \log \left\|F_{\sigma^{n}(\lambda)} \circ \cdots \circ F_{\lambda}(x)\right\|=d G_{\lambda}(x)
$$

for each $\lambda \in M$. 
Finally, pick $x_{0} \in \mathcal{A}_{\lambda_{0}}$ which by definition means that $\| F_{\sigma^{n-1}\left(\lambda_{0}\right)} \circ \cdots \circ F_{\sigma\left(\lambda_{0}\right)} \circ$ $F_{\lambda_{0}}\left(x_{0}\right) \| \leq \epsilon$ for all large $n$. Therefore, $G_{n, \lambda_{0}}\left(x_{0}\right) \leq d^{-n} \log \epsilon$ and hence $G_{\lambda_{0}}\left(x_{0}\right) \leq$ 0 . Suppose on the contrary that $G_{\lambda_{0}}\left(x_{0}\right)=0$. Note that there exists a uniform $r>0$ such that

$$
\left\|F_{\lambda}(x)\right\| \leq(1 / 2)\|x\|
$$

for all $\lambda \in M$ and $\|x\| \leq r$. This shows that the ball $B_{r}$ around the origin is contained in all the basins $\mathcal{A}_{\lambda}$. Now $G_{\lambda}(0)=-\infty$ for all $\lambda \in M$ and since $G_{\lambda_{n}} \rightarrow$ $G_{\lambda}$ locally uniformly on $\mathbb{C}^{k+1} \backslash\{0\}$ as $\lambda_{n} \rightarrow \lambda$ in $M$, it follows that there exists a large $C>0$ such that

$$
\sup _{(\lambda, x) \in M \times \partial B_{r}} G_{\lambda}(x) \leq-C .
$$

By the maximum principle, it follows that for all $\lambda \in M$,

$$
G_{\lambda}(x) \leq-C
$$

on $B_{r}$. On the other hand, the invariance property $G_{\sigma(\lambda)} \circ F_{\lambda}=d G_{\lambda}$ implies that

$$
d^{n} G_{\lambda}=G_{\sigma^{n}(\lambda)} \circ F_{\sigma^{n-1}(\lambda)} \circ \cdots \circ F_{\lambda}
$$

for all $n \geq 1$. Since we are assuming that $G_{\lambda_{0}}\left(x_{0}\right)=0$, it follows that

$$
G_{\sigma^{n}\left(\lambda_{0}\right)} \circ F_{\sigma^{n-1}\left(\lambda_{0}\right)} \circ \cdots \circ F_{\lambda_{0}}\left(x_{0}\right)=0
$$

for all $n \geq 1$ as well. But $F_{\sigma^{n-1}\left(\lambda_{0}\right)} \circ \cdots \circ F_{\sigma\left(\lambda_{0}\right)} \circ F_{\lambda_{0}}\left(x_{0}\right)$ is eventually contained in $B_{r}$ for large $n$ and this means that

$$
0=G_{\sigma^{n}\left(\lambda_{0}\right)} \circ F_{\sigma^{n-1}\left(\lambda_{0}\right)} \circ \cdots \circ F_{\lambda_{0}}\left(x_{0}\right) \leq-C
$$

by (3.3). This is a contradiction. Thus $\mathcal{A}_{\lambda} \subset\left\{G_{\lambda}<0\right\}$ for all $\lambda \in M$.

For the other inclusion, let $x \in \mathbb{C}^{k+1}$ be such that $G_{\lambda}(x)=-a$ for some $a>0$. This implies that for a given $\epsilon>0$ there exist $j_{0}$ such that

$$
-(a+\epsilon)<\frac{1}{d^{j}} \log \left\|F_{\sigma^{j-1}(\lambda)} \circ \cdots \circ F_{\lambda}(x)\right\|<-a+\epsilon
$$

for all $j \geq j_{0}$. This shows that $F_{\sigma^{j-1}(\lambda)} \circ \cdots \circ F_{\lambda}(x) \rightarrow 0$ as $j \rightarrow \infty$. Hence $x \in \mathcal{A}_{\lambda}$.

\subsection{Proof of Proposition 1.7.}

Proof. Recall that $\Omega_{\lambda}=\pi\left(\mathcal{Q}_{\lambda}\right)$ where $\mathcal{Q}_{\lambda} \subset \mathbb{C}^{k+1}$ is the collection of those points in a neighborhood of which $G_{\lambda}$ is pluriharmonic and $\Omega_{\lambda}^{\prime} \subset \mathbb{P}^{k}$ consists of those points $z \in \mathbb{P}^{k}$ in a neighborhood of which the sequence

$$
\left\{f_{\sigma^{n-1}(\lambda)} \circ \cdots \circ f_{\sigma(\lambda)} \circ f_{\lambda}\right\}_{n \geq 1}
$$

is normal, i.e., $\Omega_{\lambda}^{\prime}$ is the Fatou set. Once it is known that the basin $\mathcal{A}_{\lambda}=\left\{G_{\lambda}<0\right\}$, showing that $\Omega_{\lambda}=\Omega_{\lambda}^{\prime}$ and that each $\Omega_{\lambda}$ is in fact pseudoconvex and Kobayashi hyperbolic follows in much the same way as in 22 . Here are the main points in the proof:

Step 1. For each $\lambda \in M$, a point $p \in \Omega_{\lambda}$ if and only if there exists a neighborhood $U_{\lambda, p}$ of $p$ and a holomorphic section $s_{\lambda}: U_{\lambda, p} \rightarrow \mathbb{C}^{k+1}$ such that $s_{\lambda}\left(U_{\lambda, p}\right) \subset \partial \mathcal{A}_{\lambda}$. The choice of such a section $s_{\lambda}$ is unique upto a constant with modulus 1 . 
Suppose that $p \in \Omega_{\lambda}$. Let $U_{\lambda, p}$ be an open ball with center at $p$ that lies in a single coordinate chart with respect to the standard coordinate system of $\mathbb{P}^{k}$. Then $\pi^{-1}\left(U_{\lambda, p}\right)$ can be identified with $\mathbb{C}^{*} \times U_{\lambda, p}$ in canonical way and each point of $\pi^{-1}\left(U_{\lambda, p}\right)$ can be written as $(c, z)$. On $\pi^{-1}\left(U_{\lambda, p}\right)$, the function $G_{\lambda}$ has the form

$$
G_{\lambda}(c, z)=\log |c|+\gamma_{\lambda}(z)
$$

by (3.2). Assume that there is a section $s_{\lambda}$ such that $s_{\lambda}\left(U_{\lambda, p}\right) \subset \partial \mathcal{A}_{\lambda}$. Note that $s_{\lambda}(z)=\left(\sigma_{\lambda}(z), z\right)$ in $U_{\lambda, p}$ where $\sigma_{\lambda}$ is a non-vanishing holomorphic function on $U_{\lambda, p}$. By Proposition 1.6. $G_{\lambda} \circ s_{\lambda}=0$ on $U_{\lambda, p}$. Thus

$$
0=G_{\lambda} \circ s_{\lambda}(z)=\log \left|\sigma_{\lambda}(z)\right|+\gamma_{\lambda}(z) .
$$

Thus $\gamma_{\lambda}(z)=-\log \left|\sigma_{\lambda}(z)\right|$ is pluriharmonic on $U_{\lambda, p}$ and consequently $G_{\lambda}$ is pluriharmonic on $\pi^{-1}\left(U_{\lambda, p}\right)$ by (3.4). On the other hand, suppose that $\gamma_{\lambda}$ is pluriharmonic. Then there exists a conjugate function $\gamma_{\lambda}^{*}$ on $U_{\lambda, p}$ such that $\gamma_{\lambda}+i \gamma_{\lambda}^{*}$ is holomorphic. Define $\sigma_{\lambda}(z)=\exp \left(-\gamma_{\lambda}(z)-i \gamma_{\lambda}^{*}(z)\right)$ and $s_{\lambda}(z)=\left(\sigma_{\lambda}(z), z\right)$. Then $G_{\lambda}\left(s_{\lambda}(z)\right)=\log \left|\sigma_{\lambda}(z)\right|+\gamma_{\lambda}(z)=0$ which shows that $s_{\lambda}\left(U_{\lambda, p}\right) \subset \partial \mathcal{A}_{\lambda}$.

Step 2. $\Omega_{\lambda}=\Omega_{\lambda}^{\prime}$ for each $\lambda \in M$.

Let $p \in \Omega_{\lambda}^{\prime}$ and suppose that $U_{\lambda, p}$ is a neighborhood of $p$ on which there is a subsequence of

$$
\left\{f_{\sigma^{j-1}(\lambda)} \circ \cdots \circ f_{\lambda}\right\}_{j \geq 1}
$$

which is uniformly convergent. Without loss of generality we may assume that

$$
g_{\lambda}=\lim _{j \rightarrow \infty} f_{\sigma^{j-1}(\lambda)} \circ \cdots \circ f_{\lambda}
$$

on $U_{\lambda, p}$. By rotating the homogeneous coordinates $\left[x_{0}: x_{1}: \cdots: x_{k}\right]$ on $\mathbb{P}^{k}$, we may assume that $g_{\lambda}(p)$ avoids the hyperplane at infinity $H=\left\{x_{0}=0\right\}$ and that $g_{\lambda}(p)$ is of the form $\left[1: g_{1}: \cdots: g_{k}\right]$. Now choose an $\epsilon$ neighborhood

$$
N_{\epsilon}=\left\{\left|x_{0}\right|<\epsilon\left(\left|x_{0}\right|^{2}+\cdots+\left|x_{k}\right|^{2}\right)^{1 / 2}\right\}
$$

of $\pi^{-1}(H)$ in $\mathbb{C}^{k+1} \backslash\{0\}$ so that

$$
1>\epsilon\left(1+\left|g_{1}\right|^{2}+\cdots+\left|g_{k}\right|^{2}\right)^{1 / 2} .
$$

Clearly $g_{\lambda}(p) \notin \pi\left(N_{\epsilon}\right)$. Shrink $U_{\lambda, p}$ if needed so that

$$
f_{\sigma^{j-1}(\lambda)} \circ \cdots \circ f_{\lambda}\left(U_{\lambda, p}\right)
$$

is uniformly separated from $\pi\left(N_{\epsilon}\right)$ for sufficiently large $l$. Define

$$
s_{\lambda}(z)= \begin{cases}\log \|z\|, & \text { if } z \in N_{\epsilon}, \\ \log \left(\left|z_{0}\right| /|\epsilon|\right), & \text { if } z \in \mathbb{C}^{k+1} \backslash\left(N_{\epsilon} \cup\{0\}\right) .\end{cases}
$$

Note that $0 \leq s(z)-\log \|z\| \leq \log (1 / \epsilon)$ which implies that

$$
d^{-j} s_{\lambda}\left(f_{\sigma^{j-1}(\lambda)} \circ \cdots \circ f_{\lambda}(z)\right)
$$

converges uniformly to the Green function $G_{\lambda}$ as $j \rightarrow \infty$ on $\mathbb{C}^{k+1}$. Further, if $z \in \pi^{-1}\left(U_{\lambda, p}\right)$, then

$$
F_{\sigma^{j-1}(\lambda)} \circ \cdots \circ F_{\lambda}(z) \in \mathbb{C}^{k+1} \backslash\left(N_{\epsilon} \cup\{0\}\right) .
$$

This shows that $d^{-j} s_{\lambda}\left(f_{\sigma^{j-1}(\lambda)} \circ \cdots \circ f_{\lambda}(z)\right)$ is pluriharmonic in $\pi^{-1}\left(U_{\lambda, p}\right)$ and as a consequence the limit function $G_{\lambda}$ is also pluriharmonic in $\pi^{-1}\left(U_{\lambda, p}\right)$. Thus $p \in \Omega_{\lambda}$. 
Now pick a point $p \in \Omega_{\lambda}$. Choose a neighborhood $U_{\lambda, p}$ of $p$ and a section $s_{\lambda}: U_{\lambda, p} \rightarrow \mathbb{C}^{k+1}$ as in Step 1. Since $F_{\lambda}: \mathcal{A}_{\lambda} \rightarrow \mathcal{A}_{\sigma(\lambda)}$ is a proper map for each $\lambda$, it follows that

$$
\left(F_{\sigma^{j-1}(\lambda)} \circ \cdots \circ F_{\sigma(\lambda)} \circ F_{\lambda}\right)\left(s_{\lambda}\left(U_{\lambda, p}\right)\right) \subset \partial \mathcal{A}_{\sigma^{j}(\lambda)} .
$$

It was noted earlier that there exists a $R>0$ such that $\left\|F_{\lambda}(x)\right\| \geq 2\|x\|$ for all $\lambda$ and $\|x\| \geq R$. This shows that $\mathcal{A}_{\lambda} \subset B_{R}$ for all $\lambda \in M$, which in turn implies that the sequence

$$
\left\{\left(F_{\sigma^{j-1}(\lambda)} \circ \cdots \circ F_{\sigma(\lambda)} \circ F_{\lambda}\right) \circ s_{\lambda}\right\}_{j \geq 0}
$$

is uniformly bounded on $U_{\lambda, p}$. We may assume that it converges and let $g_{\lambda}: U_{\lambda, p} \rightarrow$ $\mathbb{C}^{k+1}$ be its limit function. Then $g_{\lambda}\left(U_{\lambda, p}\right) \subset \mathbb{C}^{k+1} \backslash\{0\}$ since all the boundaries $\partial \mathcal{A}_{\lambda}$ are at a uniform distance away from the origin; indeed, recall that there exists a uniform $r>0$ such that the ball $B_{r} \subset \mathcal{A}_{\lambda}$ for all $\lambda \in M$. Thus $\pi \circ g_{\lambda}$ is well defined and the sequence $\left\{f_{\sigma^{j-1}(\lambda)} \circ \cdots \circ f_{\sigma(\lambda)} \circ f_{\lambda}\right\}_{j \geq 0}$ converges to $\pi \circ g_{\lambda}$ uniformly on compact sets. Thus $\left\{f_{\sigma^{j-1}(\lambda)} \circ \cdots \circ f_{\sigma(\lambda)} \circ f_{\lambda}\right\}_{j \geq 0}$ is a normal family in $U_{\lambda, p}$. Hence $p \in \Omega_{\lambda}^{\prime}$.

Step 3. Each $\Omega_{\lambda}$ is pseudoconvex and Kobayashi hyperbolic.

In Lemma 2.4 of [22], Ueda proved that if $h$ is a plurisubharmonic function on $\mathbb{C}^{k}$ and

$$
\mathcal{Q}=\left\{x \in \mathbb{C}^{k}: h \text { is pluriharmonic in a neighborhood of } \mathrm{x}\right\}
$$

is non-empty, then $\mathcal{Q}$ is pseudoconvex in $\mathbb{C}^{k}$. This implies that $\Omega_{\lambda}$ is pseudoconvex for each $\lambda \in M$.

Next we want to prove that $\Omega_{\lambda}$ is Kobayashi hyperbolic for each $\lambda \in M$. A complex manifold $M$ is called Kobayashi hyperbolic if the Kobayashi pseudo distance on it is a distance. We recall some relevant facts about Kobayashi hyperbolicity which we use in the course of the following proof: (1) Any bounded domain in $\mathbb{C}^{k}$ turns out to be Kobayashi hyperbolic. (2) If $M$ is Kobayashi hyperbolic and $g: N \rightarrow M$ is injective holomorphic map, then $N$ is Kobayashi hyperbolic. (3) A manifold $M$ is hyperbolic if its covering manifold is hyperbolic.

To show that $\Omega_{\lambda}$ is Kobayashi hyperbolic, it suffices to prove that each component $U$ of $\Omega_{\lambda}$ is Kobayashi hyperbolic. For a point $p$ in $U$ choose $U_{\lambda, p}$ and $s_{\lambda}$ as in Step 1. Then $s_{\lambda}$ can be analytically continued to $U$. This analytic continuation of $s_{\lambda}$ gives a holomorphic map $\tilde{s}_{\lambda}: \widetilde{U} \rightarrow \mathbb{C}^{k+1}$ satisfying $\pi \circ \tilde{s}_{\lambda}=p$ where $\widetilde{U}$ is a covering of $U$ and $p: \widetilde{U} \rightarrow U$ is the corresponding covering map. Note that there exists a uniform $R>0$ such that $\left\|F_{\lambda}(z)\right\| \geq 2\|z\|$ for all $\lambda \in M$ and for all $z \in \mathbb{C}^{k+1}$ with $\|z\| \geq R$. Thus $\mathcal{A}_{\lambda} \subset B(0, R)$ and $\tilde{s}_{\lambda}(\widetilde{U}) \subset B(0,2 R)$. Since $\tilde{s}_{\lambda}$ is injective and $B(0,2 R)$ is Kobayashi hyperbolic in $\mathbb{C}^{k+1}$, it follows that $\widetilde{U}$ is Kobayashi hyperbolic. Hence $U$ is Kobayashi hyperbolic.

\section{ACKNOWLEDGMENT}

The first named author would like to thank G. Buzzard and M. Jonsson for their helpful comments in an earlier version of this paper. 


\section{REFERENCES}

[1] E. Bedford and J. Smillie, Polynomial diffeomorphisms of $\mathbf{C}^{2}$ : currents, equilibrium measure and hyperbolicity, Invent. Math. 103 (1991), no. 1, 69-99, DOI 10.1007/BF01239509. MR 1079840 (92a:32035)

[2] E. Bedford and J. Smillie, Polynomial diffeomorphisms of $\mathbf{C}^{2}$. II. Stable manifolds and recurrence, J. Amer. Math. Soc. 4 (1991), no. 4, 657-679, DOI 10.2307/2939284. MR.1115786 (92m:32048)

[3] E. Bedford and J. Smillie, Polynomial diffeomorphisms of $\mathbf{C}^{2}$. III. Ergodicity, exponents and entropy of the equilibrium measure, Math. Ann. 294 (1992), no. 3, 395-420, DOI 10.1007/BF01934331. MR1188127 (93k:32062)

[4] D. Coman and J. E. Fornæss, Green's functions for irregular quadratic polynomial automorphisms of $\mathbf{C}^{3}$, Michigan Math. J. 46 (1999), no. 3, 419-459, DOI 10.1307/mmj/1030132474. MR.1721516(2000i:32030)

[5] D. Coman and V. Guedj, Invariant currents and dynamical Lelong numbers, J. Geom. Anal. 14 (2004), no. 2, 199-213, DOI 10.1007/BF02922068. MR2051683 (2005d:32056)

[6] H. de Thélin, Endomorphismes pseudo-aléatoires dans les espaces projectifs I (French, with English and French summaries), Manuscripta Math. 142 (2013), no. 3-4, 347-367, DOI 10.1007/s00229-012-0603-9. MR3117166

[7] H. De Thélin, Endomorphismes pseudo-aléatoires dans les espaces projectifs II (French, with English and French summaries), J. Geom. Anal. 25 (2015), no. 1, 204-225, DOI 10.1007/s12220-013-9422-9. MR3299276

[8] T. C. Dinh, N. Sibony, Rigidity of Julia sets for Hénon type maps, Proceedings of the 20082011 Summer Institute at Bedlewo. Modern Dynamics and its Interaction with Analysis, Geometry and Number Theory, To appear.

[9] C. Favre and M. Jonsson, Dynamical compactifications of $\mathbf{C}^{2}$, Ann. of Math. (2) 173 (2011), no. 1, 211-248, DOI 10.4007/annals.2011.173.1.6. MR2753603 (2012d:32025)

[10] S. Friedland and J. Milnor, Dynamical properties of plane polynomial automorphisms, Ergodic Theory Dynam. Systems 9 (1989), no. 1, 67-99, DOI 10.1017/S014338570000482X. MR.991490 (90f:58163)

[11] J. E. Fornæss and N. Sibony, Complex Hénon mappings in $\mathbf{C}^{2}$ and Fatou-Bieberbach domains, Duke Math. J. 65 (1992), no. 2, 345-380, DOI 10.1215/S0012-7094-92-06515-X. MR.1150591 (93d:32040)

[12] J. E. Fornæss and $\mathrm{H}$. Wu, Classification of degree 2 polynomial automorphisms of $\mathbf{C}^{3}$, Publ. Mat. 42 (1998), no. 1, 195-210, DOI 10.5565/PUBLMAT_42198_10. MR1628170(99e:14015)

[13] V. Guedj, Courants extrémaux et dynamique complexe (French, with English and French summaries), Ann. Sci. École Norm. Sup. (4) 38 (2005), no. 3, 407-426, DOI 10.1016/j.ansens.2005.02.001. MR.2166340 (2006k:32070)

[14] V. Guedj and N. Sibony, Dynamics of polynomial automorphisms of $\mathbf{C}^{k}$, Ark. Mat. 40 (2002), no. 2, 207-243, DOI 10.1007/BF02384535. MR.1948064 (2004b:32029)

[15] J. H. Hubbard and P. Papadopol, Superattractive fixed points in $\mathbf{C}^{n}$, Indiana Univ. Math. J. 43 (1994), no. 1, 321-365, DOI 10.1512/iumj.1994.43.43014. MR.1275463 (95e:32025)

[16] M. Jonsson, Dynamics of polynomial skew products on $\mathbf{C}^{2}$, Math. Ann. 314 (1999), no. 3, 403-447, DOI 10.1007/s002080050301. MR.1704543 (2000f:32025)

[17] M. Jonsson, Ergodic properties of fibered rational maps, Ark. Mat. 38 (2000), no. 2, 281-317, DOI 10.1007/BF02384321. MR1785403 (2002k:37073)

[18] H. Peters, Non-autonomous dynamics in $\mathbb{P}^{k}$, Ergodic Theory Dynam. Systems 25 (2005), no. 4, 1295-1304, DOI 10.1017/S0143385704000987. MR2158406 (2006b:37085)

[19] H. Peters and E. F. Wold, Non-autonomous basins of attraction and their boundaries, J. Geom. Anal. 15 (2005), no. 1, 123-136, DOI 10.1007/BF02921861. MR2132268(2006a:37035)

[20] N. Sibony, Dynamique des applications rationnelles de $\mathbf{P}^{k}$ (French, with English and French summaries), Dynamique et géométrie complexes (Lyon, 1997), Panor. Synthèses, vol. 8, Soc. Math. France, Paris, 1999, pp. ix-x, xi-xii, 97-185. MR1760844 (2001e:32026)

[21] J. Smillie, The entropy of polynomial diffeomorphisms of $\mathbf{C}^{2}$, Ergodic Theory Dynam. Systems 10 (1990), no. 4, 823-827, DOI 10.1017/S0143385700005927. MR1091429 (92b:58131)

[22] T. Ueda, Fatou sets in complex dynamics on projective spaces, J. Math. Soc. Japan 46 (1994), no. 3, 545-555, DOI 10.2969/jmsj/04630545. MR1276837 (95d:32030) 
[23] S. Morosawa, Y. Nishimura, M. Taniguchi, and T. Ueda, Holomorphic dynamics, Cambridge Studies in Advanced Mathematics, vol. 66, Cambridge University Press, Cambridge, 2000. Translated from the 1995 Japanese original and revised by the authors. MR.1747010 (2002c:37064)

[24] Y. Yomdin, Volume growth and entropy, Israel J. Math. 57 (1987), no. 3, 285-300, DOI 10.1007/BF02766215. MR889979 (90g:58008)

[25] P. Walters, An introduction to ergodic theory, Graduate Texts in Mathematics, vol. 79, Springer-Verlag, New York-Berlin, 1982. MR648108 (84e:28017)

Department of Mathematics, Indian Institute of Science, Bangalore 560 012, India

E-mail address: ratna10@math.iisc.ernet.in

Department of Mathematics, Indian Institute of Science, Bangalore 560 012, India

E-mail address: kverma@math.iisc.ernet.in 